\title{
Graph limits of random unlabelled $\boldsymbol{k}$-trees
}

\author{
Emma Yu Jin ${ }^{1, \dagger}$ and Benedikt Stufler ${ }^{2 \ddagger}$ \\ ${ }^{1}$ Fakultät für Mathematik, Universität Wien, Oskar-Morgenstern Platz 1, 1090 Vienna, Austria and ${ }^{2}$ Institut für Mathematik, \\ Universität München, Theresienstr. 39, D-80333 Munich, Germany \\ *Corresponding author. Email: yu.jin@univie.ac.at
}

(Received 18 June 2018; revised 1 March 2020; accepted 12 March 2020; first published online 18 May 2020)

\begin{abstract}
We study random unlabelled $k$-trees by combining the colouring approach by Gainer-Dewar and Gessel (2014) with the cycle-pointing method by Bodirsky, Fusy, Kang and Vigerske (2011). Our main applications are Gromov-Hausdorff-Prokhorov and Benjamini-Schramm limits that describe their asymptotic geometric shape on a global and local scale as the number of $(k+1)$-cliques tends to infinity.
\end{abstract}

2020 MSC Codes: Primary 60C05; Secondary 05C80, 05A16

\section{Introduction}

A $k$-tree, or $k$-dimensional tree, may be defined recursively: it is either a complete graph on $k$ vertices or a graph obtained from a smaller $k$-tree by adjoining a new vertex together with $k$ edges, connecting it to a $k$-clique of the smaller $k$-tree. This concept naturally generalizes trees that correspond to the special case $k=1$. We may distinguish $k$-trees whose vertices are labelled by elements of some fixed set, and unlabelled $k$-trees, which are $k$-trees considered up to graph isomorphism. It is customary to index $k$-trees by their number of $(k+1)$-cliques, which are called hedra in this context. Thus, the number of vertices in a $k$-tree having $n$ hedra is given by $n+k$. For instance, there are five different 2 -trees with four hedra: see Figure 1 . A $k$-clique in a $k$-tree is usually called a front.

The counting problem of the class of $k$-trees has a long history. The number of labelled $k$ trees of any fixed size was obtained by Beineke, Pippert, Moon and Foata $[2,11,20]$, and the enumeration of unlabelled 1-trees is a classical result attributed to Otter [21]. Subsequently, unlabelled 2-trees were counted by Harary and Palmer [15, 16] as well as Fowler, Gessel, Labelle and Leroux [12] using the dissimilarity characteristic theorem. However, counting general unlabelled $k$-trees $(k \geqslant 3)$ was a long-standing open problem, which was only recently solved by Gainer-Dewar [13] using $\Gamma$-species. A simpler proof that combines front-colourings with hedronlabellings was later discovered by Gainer-Dewar and Gessel [14]. The advantage of this approach is that front-colouring breaks the symmetry of unlabelled $k$-trees and avoids the use of compatible cyclic orientation of each $(k+1)$-clique in a $k$-tree. Based on the simplified generating functions

\footnotetext{
${ }^{\dagger}$ The first author was supported by FWF-MOST (Austrian-Taiwanese) project I 2309-N35 and FWF Project P 32305.

₹The second author gratefully acknowledges support by the German Research Foundation DFG, STU 679/1-1 and the Swiss National Science Foundation grant number 200020_172515.

(C) The Author(s), 2020. Published by Cambridge University Press. This is an Open Access article, distributed under the terms of the Creative Commons Attribution licence (http://creativecommons.org/licenses/by/4.0/), which permits unrestricted re-use, distribution, and reproduction in any medium, provided the original work is properly cited.
} 

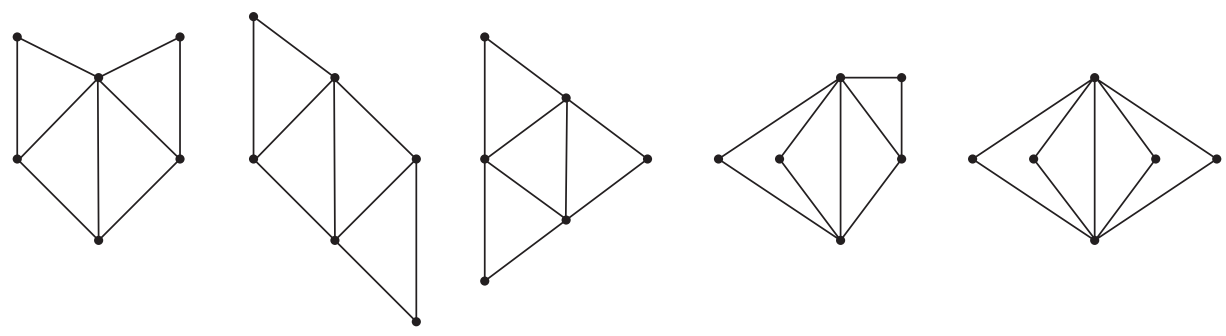

Figure 1. All unlabelled 2-trees with four hedra (triangles).

from [14], Drmota and the first author [7] have undertaken a systematic asymptotic analysis of unlabelled $k$-trees using singularity analysis.

In the present work we establish a substraction-free combinatorial decomposition of unlabelled $k$-trees (see (5.8)). This is motivated by the fact that all prior analyses of unlabelled $k$-trees (for $k \geqslant 2$ ) are based on double-counting arguments in the form of a dissymmetry theorem. The substraction operation in the resulting functional equations of generating series severely complicates any follow-up probabilistic analyses, since the corresponding Boltzmann sampling procedures have to employ a costly rejection process. In order to tackle this, we combine the front-colouring method by Gainer-Dewar and Gessel [14] with the framework of unlabelled $\mathcal{R}$-enriched trees by the second author [27] and the cycle-pointing method by Bodirsky, Fusy, Kang and Vigerske [5]. The latter approach is based on the idea of considering graphs marked at a cyclic permutation of some of the vertices, such that the cycle appears in at least one automorphism. A similar approach was also used in [28] for unlabelled trees with vertex-degree restrictions, and the present work intersects with this paper precisely for the case of unrestricted 1-trees. The decomposition in [28] is not suitable for $k$-trees if $k \geqslant 2$. Conversely, the decomposition established in the present work is not suitable for accommodating vertex-degree restrictions of 1-trees.

With the help of a rejection-free sampling procedure, we conduct a probabilistic study of the random $k$-tree $\mathrm{U}_{n}=\mathrm{U}_{n, k}$ chosen uniformly at random among all unlabelled $k$-trees with $n$ hedra. Our main results are a Gromov-Hausdorff-Prokhorov scaling limit (Theorem 2.1) and a Benjamini-Schramm limit (Theorem 2.3) for $\bigcup_{n}$ as $n$ tends to infinity, which will be presented in detail in Section 2 along with a brief probabilistic background on the graph limits.

The rest of this paper is organized as follows. In Sections 3 and 4 we review some previous results on unlabelled $k$-trees, namely the front-colouring approach and the study of unlabelled $\mathcal{R}$-enriched trees. In Section 5 we develop a substraction-free decomposition of unlabelled $k$-trees via the cycle-pointing method, which enables us to prove our main results in Section 6.

Notation. Throughout, we set $[n]=\{1,2, \ldots, n\}$ for all integers $n \geqslant 0$. The random variables appearing in this paper are either canonical or defined on a common probability space whose measure we denote by $\mathbb{P}$. All unspecified limits are taken as $n$ becomes large. We let $\stackrel{d}{\longrightarrow}$ denote convergence in distribution, and denote equality in distribution by $\stackrel{d}{=}$. The total variation distance of measures and random variables is denoted by $d_{\mathrm{TV}}$. That is, if $X$ and $Y$ are random variables with values in a common metric space, then

$$
d_{\mathrm{TV}}(X, Y)=\sup _{A}|\mathbb{P}(X \in A)-\mathbb{P}(Y \in A)|,
$$

with the index $A$ ranging over all Borel-measurable subsets of the target space. An event (that depends on $n$ ) holds with high probability if its probability tends to 1 as $n$ tends to infinity. We say it is exponentially unlikely if there are constants $C, c>0$ such that its probability is bounded by $C \exp (-c n)$ for all $n$. Likewise, we say it is exponentially likely if its complement is exponentially 
unlikely. For any integer $n \geqslant 0$ and any power series $f(z)$, we let $\left[z^{n}\right] f(z)$ denote the coefficient of $z^{n}$ in $f(z)$.

\section{Probabilistic background and main results}

\subsection{Scaling limits: what a random graph looks like when viewed from far away}

A precise statement of the scaling limits of random unlabelled $k$-trees is established in Theorem 2.1. The reader familiar with the scaling limits may skip Sections 2.1.1, 2.1.2 and 2.1.3.

\subsubsection{Gromov-Hausdorff-Prokhorov convergence}

We briefly recall some probabilistic background following Miermont [19, Section 6]. Suppose that $X=\left(X, d_{X}\right)$ and $Y=\left(Y, d_{Y}\right)$ are compact metric spaces, and the diameter of a space $X$ is denoted by

$$
\mathrm{D}(X)=\sup _{x, x^{\prime} \in X} d_{X}\left(x, x^{\prime}\right)
$$

A correspondence between the spaces $X$ and $Y$ is a subset $R \subset X \times Y$ such that for any $x \in X$ there is a point $y \in Y$ with $(x, y) \in R$, and conversely for any $y \in Y$ there is a point $x \in X$ with $(x, y) \in R$. We let $\mathcal{C}(X, Y)$ denote the collection of all correspondences between $X$ and $Y$. We also define the subset $\mathcal{C}_{c}(X, Y) \subset \mathcal{C}(X, Y)$ of all correspondences that are compact. The distortion of the correspondence $R$ may be defined as the supremum

$$
\operatorname{dis}(R)=\sup \left\{\left|d_{X}\left(x_{1}, x_{2}\right)-d_{Y}\left(y_{1}, y_{2}\right)\right| \mid\left(x_{1}, y_{1}\right),\left(x_{2}, y_{2}\right) \in R\right\} .
$$

Suppose that we are additionally given a Borel probability measure $\mu_{X}$ on $X$ and a Borel probability measure $\mu_{Y}$ on $Y$. A coupling of $\mu_{X}$ and $\mu_{Y}$ is a probability measure $v$ on the product space $X \times Y$ such that the pushforward measures along the restrictions $\pi_{X}: X \times Y \rightarrow X$ and $\pi_{Y}: X \times Y \rightarrow Y$ are equal to $\mu_{X}$ and $\mu_{Y}$. That is, $v(A \times Y)=\mu_{X}(A)$ for all Borel-measurable subsets $A \subset X$, and $v(X \times B)=\mu_{Y}(B)$ for all Borel-measurable subsets $B \subset Y$. We let $\mathcal{M}(X, Y)$ denote the collection of such couplings $\nu$. Informally, a coupling should be viewed as a clever way to jointly construct a $\mu_{X}$-distributed random variable together with a $\mu_{Y}$-distributed random variable. The Gromov-Hausdorff-Prokhorov distance between the measured metric spaces $\left(X, d_{X}, \mu_{X}\right)$ and $\left(Y, d_{Y}, \mu_{Y}\right)$ may be defined by

$$
\begin{aligned}
& d_{\mathrm{GHP}}\left(\left(X, d_{X}, \mu_{X}\right),\left(Y, d_{Y}, \mu_{Y}\right)\right) \\
& \quad=\inf \left\{\epsilon>0 \mid \exists R \in \mathcal{C}_{c}(X, Y), v \in \mathcal{M}(X, Y): \operatorname{dis}(R) \leqslant 2 \epsilon, v(R)>1-\epsilon\right\} .
\end{aligned}
$$

The collection $\mathbb{K}$ of (representatives of isometry classes of) compact measured metric spaces equipped with the Gromov-Hausdorff-Prokhorov metric is a Polish space, meaning that standard probabilistic notions like distributional convergence of random elements apply.

\subsubsection{Consequences of Gromov-Hausdorff-Prokhorov convergence}

Suppose that $\left(\mathrm{X}_{n}, d_{\mathrm{X}_{n}}, \mu_{\mathrm{X}_{n}}\right)$ is a sequence of random compact measured metric spaces satisfying

$\left(\mathrm{X}_{n}, d_{\mathrm{X}_{n}}, \mu_{\mathrm{X}_{n}}\right) \stackrel{d}{\longrightarrow}\left(\mathrm{X}, d_{\mathrm{X}}, \mu_{\mathrm{X}}\right)$ in the Gromov-Hausdorff-Prokhorov sense for some random compact measured metric space $\left(\mathrm{X}, d_{\mathrm{X}}, \mu_{\mathrm{X}}\right)$. The well-known continuous mapping theorem ensures that, for any Polish space $Y$ and any function $f: \mathbb{K} \rightarrow Y$ whose points of continuity $\Omega \subset \mathbb{K}$ satisfy $\mathbb{P}\left(\left(\mathrm{X}, d_{\mathrm{X}}, \mu_{\mathrm{X}}\right) \in \Omega\right)=1$,

$$
f\left(\mathrm{X}_{n}, d_{\mathrm{X}_{n}}, \mu_{\mathrm{X}_{n}}\right) \stackrel{d}{\longrightarrow} f\left(\mathrm{X}, d_{\mathrm{X}}, \mu_{\mathrm{X}}\right) .
$$


In particular, this applies to the diameter $\mathrm{D}(\cdot)$ which is continuous on $\mathbb{K}$, yielding

$$
\mathrm{D}\left(\mathrm{X}_{n}, d_{\mathrm{x}_{n}}, \mu_{\mathrm{X}_{n}}\right) \stackrel{d}{\longrightarrow} \mathrm{D}\left(\mathrm{X}, d_{\mathrm{X}}, \mu_{\mathrm{X}}\right) \text {. }
$$

Let $r \geqslant 2$ denote a fixed integer and suppose that the compact metric space $\left(X, d_{X}\right)$ additionally has $r$ distinguished compact subsets $C_{1}, \ldots, C_{r}$, resulting in an $r$-pointed space $X^{\bullet}$. Likewise, let $D_{1}, \ldots, D_{r}$ denote $r$ distinguished compact subsets of the space $Y$, resulting in an $r$-pointed space $Y^{\bullet^{r}}$. We let $\mathcal{C}^{\bullet}\left(X^{{ }^{r}}, Y^{\bullet^{r}}\right)$ denote the collection of correspondences $R \in \mathcal{C}(X, Y)$ that additionally satisfy $C_{i} \times D_{i} \in \mathcal{C}\left(C_{i}, D_{i}\right)$ for all $1 \leqslant i \leqslant r$. The marked Gromov-Hausdorff distance between these marked spaces is defined by

$$
d_{\mathrm{GH}}^{\bullet}\left(X^{\bullet^{r}}, Y^{\bullet^{r}}\right)=\max \left(\frac{1}{2} \inf _{R \in \mathcal{C}_{c}^{\bullet}(X, Y)} \operatorname{dis}(R)\right) .
$$

This makes the collection of (representatives of mark-preserving isometry classes of) marked compact metric spaces a Polish space, hence standard notions of convergence of random elements apply.

Now suppose as before that $\left(\mathrm{X}_{n}, d_{\mathrm{X}_{n}}, \mu_{\mathrm{X}_{n}}\right) \stackrel{d}{\longrightarrow}\left(\mathrm{X}, d_{\mathrm{X}}, \mu_{\mathrm{X}}\right)$ in the Gromov-HausdorffProkhorov sense. Consider the space $\mathrm{X}_{n}^{\boldsymbol{r}^{r}}$ obtained by marking $r$ independently $\mu_{\mathrm{X}_{n}}$-distributed points $\left(x_{i, n}\right)_{1 \leqslant i \leqslant r}$ of $\mathrm{X}_{n}$. Likewise, let $\mathrm{X}^{\bullet}{ }^{r}$ be marked at independent $\mu_{\mathrm{X}}$-distributed points $\left(x_{i}\right)_{1 \leqslant i \leqslant r}$. It was shown in [19, Proposition 10] that the distributional convergence in the Gromov-Hausdorff-Prokhorov sense entails that

$$
\mathrm{X}_{n}^{\mathbf{\bullet}^{r}} \stackrel{d}{\longrightarrow} \mathrm{X}^{\boldsymbol{\bullet}^{r}}
$$

in the marked Gromov-Hausdorff sense. By the continuous mapping theorem, this implies

$$
\left(d_{x_{n}}\left(x_{i, n}, x_{j, n}\right)\right)_{1 \leqslant i, j \leqslant r} \stackrel{d}{\longrightarrow}\left(d_{x}\left(x_{i}, x_{j}\right)\right)_{1 \leqslant i, j \leqslant r} .
$$

\subsubsection{The Brownian tree}

A famous and universal distributional limit object is Aldous's Brownian tree $\left(\mathcal{T}_{\mathrm{e}}, d_{\mathcal{T}_{\mathrm{e}}}, \mu_{\mathcal{T}_{\mathrm{e}}}\right)$. The construction is as follows. Consider a Brownian excursion $\left(\mathbf{e}_{t}: 0 \leqslant t \leqslant 1\right)$ of duration 1 . A possible construction of this continuous stochastic process is to start with a Brownian motion (or Wiener process) $(B(t): t \geqslant 0)$ and consider the last time $\tau_{-}$that $B(t)$ hits zero before time $t=1$, and the first time $\tau_{+}$it hits zero after time $t=1$. We may then set

$$
\mathbf{e}_{t}=\frac{\left|B\left(\tau_{-}(1-t)+\tau_{+} t\right)\right|}{\sqrt{\tau_{+}-\tau_{-}}} \text {for } 0 \leqslant t \leqslant 1 .
$$

With probability 1 this is a well-defined continuous function. For all $x, y \in[0,1]$ with $x \leqslant y$, we set

$$
\bar{d}(x, y)=\mathbf{e}_{x}+\mathbf{e}_{y}-2 \inf _{x \leqslant t \leqslant y} \mathbf{e}_{t} .
$$

If $x>y$ we set $\bar{d}(x, y):=\bar{d}(y, x)$. Topologically, the Brownian tree $\mathcal{T}_{\mathbf{e}}$ is defined as a random quotient metric space $\mathcal{T}_{\mathbf{e}}=[0,1] / \sim$ with the equivalence relation $\sim$ defined by

$$
x \sim y \quad \text { if and only if } \bar{d}(x, y)=0 .
$$

The distance $\bar{d}$ is lifted to a distance $d_{\mathcal{T}_{\mathrm{e}}}$ along the canonical surjection $\pi:[0,1] \rightarrow[0,1] / \sim$. The Borel probability measure on $\mathcal{T}_{\mathbf{e}}$ is defined as the pushforward of the Lebesgue measure $\lambda$ on the unit interval $[0,1]$. That is, for any Borel-measurable subset $A \subset \mathcal{T}_{\mathbf{e}}$ we set $\mu_{\mathcal{T}_{\mathbf{e}}}(A):=\lambda\left(\pi^{-1}(A)\right)$. By (2.4), the diameter $\mathrm{D}\left(\mathcal{T}_{\mathbf{e}}\right)$ admits the expression

$$
\mathrm{D}\left(\mathcal{T}_{\mathrm{e}}\right) \stackrel{d}{=} \sup _{0 \leqslant t_{1} \leqslant t_{2} \leqslant 1}\left(\mathrm{e}\left(t_{1}\right)+\mathrm{e}\left(t_{2}\right)-2 \inf _{t_{1} \leqslant t \leqslant t_{2}} \mathrm{e}(t)\right) .
$$


If we sample two points $v^{1}$ and $v^{2}$ of the Brownian tree $\mathcal{T}_{\mathbf{e}}$ independently according to the measure $\mu_{\mathcal{T}_{\mathrm{e}}}$, then by construction

$$
d_{\mathcal{T}_{\mathbf{e}}}\left(v^{1}, v^{2}\right) \stackrel{d}{=} \bar{d}\left(x^{1}, x^{2}\right)
$$

with $x^{1}$ and $x^{2}$ denoting two independent and uniformly selected points of the unit interval. The Brownian tree is known to satisfy a re-rooting invariance as stated by Aldous [1, equation (20)], meaning that the coset $\overline{0}$ of $0 \in[0,1]$ satisfies $\left(\mathcal{T}_{\mathbf{e}}, v^{1}\right) \stackrel{d}{=}\left(\mathcal{T}_{\mathbf{e}}, \overline{0}\right)$. Hence

$$
d_{\mathcal{T}_{\mathbf{e}}}\left(v^{1}, v^{2}\right) \stackrel{d}{=} \bar{d}\left(0, x^{1}\right)=\mathbf{e}_{x^{1}} .
$$

It is a well-known fact (see e.g. [9, Proposition 3.4]) that the standardized Brownian excursion evaluated at a uniformly and independently selected point of the unit interval follows the distribution

$$
\mathbb{P}\left(\mathbf{e}_{x^{1}} \in A\right)=\int_{A} 4 s \exp \left(-2 s^{2}\right) \mathrm{d} s
$$

for $A$ a Borel subset of $\left[0, \infty\right.$ [. Hence $2 \mathbf{e}_{x^{1}}$ follows the Rayleigh distribution with probability density $s \exp \left(-s^{2} / 2\right)$.

Now we are ready to state our first main result.

\subsubsection{Scaling limits of random unlabelled $k$-trees}

Our first main result establishes the Brownian tree $\left(\mathcal{T}_{\mathrm{e}}, d_{\mathcal{T}_{\mathrm{e}}}, \mu_{\mathcal{T}_{\mathrm{e}}}\right)$ as the Gromov-HausdorffProkhorov scaling limit of the random unlabelled $k$-tree $\cup_{n}$.

Theorem 2.1 Let $\mu_{n}$ denote the uniform measure on the set of vertices of $U_{n}$. Then there is a constant $c_{k}>0$ such that

$$
\left(\mathrm{U}_{n}, c_{k} n^{-1 / 2} d_{\mathrm{U}_{n}}, \mu_{n}\right) \stackrel{d}{\longrightarrow}\left(\mathcal{T}_{\mathrm{e}}, d_{\mathcal{T}_{\mathrm{e}}}, \mu_{\mathcal{T}_{\mathrm{e}}}\right)
$$

in the Gromov-Hausdorff-Prokhorov sense.

The scaling constant is given by

$$
c_{k}=k H_{k} \sqrt{1+k \sum_{i=2}^{\infty} \bar{B}_{1^{k}}^{\prime}\left(\rho_{k}^{i}\right) \rho_{k}^{i}} .
$$

Here $H_{k}=\sum_{i=1}^{k} i^{-1}$ denotes the $k$ th harmonic number. $\bar{B}_{1^{k}}(z)$ is the unique power series satisfying

$$
\bar{B}_{1^{k}}(z)=z \exp \left(k \sum_{i=1}^{\infty} \frac{\bar{B}_{1^{k}}\left(z^{i}\right)}{i}\right),
$$

and $\rho_{k}$ denotes its radius of convergence. See Table 1 for numerical approximations up to $k=10$. $^{*}$ It follows from [7, Theorem 3] that

$$
\rho_{k}=\frac{1}{e k}-\frac{1}{2 e^{3} k^{2}}+O\left(\frac{1}{k^{3}}\right) \text { and } k \sum_{i=2}^{\infty} \bar{B}_{1^{k}}^{\prime}\left(\rho_{k}^{i}\right) \rho_{k}^{i}=O\left(\frac{1}{k}\right)
$$

\footnotetext{
*The numerical approximation of the constant $\rho_{k}$ in Table 1 was done by taking $m:=30$, calculating a truncation $\bar{B}_{1 k}^{[m]}(z)$ up to order $m$ of $\bar{B}_{1^{k}}(z)$ via the recursive relation derived from equation (4.1), and numerically solving the truncated system $x \exp \left(k \sum_{i=2}^{m} i^{-1} \bar{B}_{1^{k}}^{[m]}\left(x^{i}\right)\right)=1 /(e k)$.
} 
Table 1. Numerical approximations of constants for unlabelled $k$-trees.

\begin{tabular}{lccc}
\hline \hline$k$ & $c_{k}$ & $\rho_{k}$ & $c_{k} /\left(k H_{k}\right)$ \\
\hline 1 & 1.102725 & 0.338321 & 1.102725 \\
2 & 3.126190 & 0.177099 & 1.042063 \\
3 & 5.643857 & 0.119674 & 1.026155 \\
4 & 8.491071 & 0.090334 & 1.018928 \\
5 & 11.585821 & 0.072539 & 1.014816 \\
6 & 14.878854 & 0.060597 & 1.012166 \\
7 & 18.337291 & 0.052031 & 1.010319 \\
8 & 21.937615 & 0.045585 & 1.008957 \\
9 & 25.662173 & 0.040561 & 1.007912 \\
10 & 29.497218 & 0.036533 & 1.007085 \\
\hline \hline
\end{tabular}

as $k$ becomes large, yielding

$$
c_{k}=\left(1+O\left(k^{-1}\right)\right) k H_{k}
$$

The diameter $\mathrm{D}(\cdot)$ is a Gromov-Hausdorff continuous function. Hence, using (2.2) and (2.5), Theorem 2.1 implies that

$$
c_{k} n^{-1 / 2} \mathrm{D}\left(\mathrm{U}_{n}\right) \stackrel{d}{\longrightarrow} \mathrm{D}\left(\mathcal{T}_{\mathrm{e}}\right) \stackrel{d}{=} \sup _{0 \leqslant t_{1} \leqslant t_{2} \leqslant 1}\left(\mathrm{e}\left(t_{1}\right)+\mathrm{e}\left(t_{2}\right)-2 \inf _{t_{1} \leqslant t \leqslant t_{2}} \mathrm{e}(t)\right),
$$

with $\mathrm{e}=\left(\mathrm{e}_{t}\right)_{0 \leqslant t \leqslant 1}$ denoting the Brownian excursion of length 1 . Let $v_{n}^{1}$ and $v_{n}^{2}$ denote two independently and uniformly selected vertices of $U_{n}$. Using (2.3), (2.6) and (2.7), the GromovHausdorff-Prokhorov convergence of Theorem 2.1 implies that

$$
2 c_{k} d_{\cup_{n}}\left(v_{n}^{1}, v_{n}^{2}\right) / \sqrt{n} \stackrel{d}{\longrightarrow} \text { Rayleigh }(1),
$$

with the limiting Rayleigh(1) distribution having density $x \exp \left(-x^{2} / 2\right), x \geqslant 0$. In fact, it also implies a scaling limit for the vector of pairwise distances for any finite fixed number of vertices that are uniformly and independently sampled.

It is important to keep in mind that in the present work we treat unlabelled $k$-trees, whose study is severely complicated by the presence of symmetries. Our results parallel a list of properties of random labelled $k$-trees, but do not encompass them and are not encompassed by them. The Rayleigh distribution has been observed to arise as scaling limit of the distance of independent random vertices in random labelled $k$-trees by Darrasse and Soria [6], but the scaling constant of (2.10) differs from the labelled case. Drmota and both authors [8] gave a scaling limit for random labelled $k$-trees, of course also with a different scaling constant.

In order to deduce convergence of the moments of $\mathrm{D}\left(\mathrm{U}_{n}\right)$ and $d_{\mathrm{U}_{n}}\left(v_{n}^{1}, v_{n}^{2}\right)$, it is sufficient to verify $p$-uniform integrability of the diameter $\mathrm{D}\left(\mathrm{U}_{n}\right)$ for arbitrarily large integers $p \geqslant 1$. For this it suffices to show that for each $p \geqslant 1$ the $\mathbb{L}^{p}$-norm of the rescaled diameter $\mathrm{D}\left(\mathrm{U}_{n}\right) / \sqrt{n}$ remains bounded as $n \rightarrow \infty$. And this is is ensured by the following sharp tail bound, which will be proved in Section 6.

Theorem 2.2 There are constants $C, c>0$ such that, for all $n \geqslant 1$ and $x \geqslant 0$,

$$
\mathbb{P}\left(\mathrm{D}\left(\mathrm{U}_{n}\right) \geqslant x\right) \leqslant C \exp \left(-c x^{2} / n\right) .
$$


This entails that for any fixed integer $p \geqslant 1$ we obtain

$$
\mathbb{E}\left[\mathrm{D}\left(\mathrm{U}_{n}\right)^{p}\right] \sim c_{k}^{-p} n^{p / 2} \mathbb{E}\left[\mathrm{D}\left(\mathcal{T}_{\mathrm{e}}\right)^{p}\right] \text { and } \mathbb{E}\left[d_{\mathrm{U}_{n}}\left(v_{n}^{1}, v_{n}^{2}\right)^{p}\right] \sim n^{p / 2} 2^{-p / 2} c_{k}^{-p} \Gamma(1+p / 2)
$$

as $n$ becomes large. The moments of the diameter of $\mathcal{T}_{\mathrm{e}}$ are known and given by

$$
\begin{aligned}
\mathbb{E}\left[\mathrm{D}\left(\mathcal{T}_{\mathrm{e}}\right)\right] & =\frac{4}{3} \sqrt{\pi / 2}, \quad \mathbb{E}\left[\mathrm{D}\left(\mathcal{T}_{\mathrm{e}}\right)^{2}\right]=\frac{2}{3}\left(1+\frac{\pi^{2}}{3}\right), \quad \mathbb{E}\left[\mathrm{D}\left(\mathcal{T}_{\mathrm{e}}\right)^{3}\right]=2 \sqrt{2 \pi}, \\
\mathbb{E}\left[\mathrm{D}\left(\mathcal{T}_{\mathrm{e}}\right)^{k}\right] & =\frac{2^{k / 2}}{3} k(k-1)(k-3) \Gamma(k / 2)(\zeta(k-2)-\zeta(k)) \quad \text { for } k \geqslant 4 .
\end{aligned}
$$

Here $\zeta$ refers to the Riemann's zeta function, and $\Gamma$ to Euler's gamma function. See [1, Section 3.4] and $[25$, Section 1.1].

\subsection{Benjamini-Schramm limits: zooming in on a random vertex in a random graph}

A precise statement of the Benjamini-Schramm limits of random unlabelled $k$-trees is established in Theorem 2.3. The reader familiar with the local limits may skip Section 2.2.1.

\subsubsection{Local convergence}

Consider two connected, rooted and locally finite graphs $G^{\bullet}=\left(G, v_{G}\right)$ and $H^{\bullet}=\left(H, v_{H}\right)$. For any integer $k \geqslant 0$ we may define the $k$-neighbourhood $U_{k}\left(G^{\bullet}\right)$ as the vertex-rooted subgraph of $G^{\bullet}$ induced by all vertices with distance at most $k$ from $v_{G}$. We construct the distance

$$
d\left(G^{\bullet}, H^{\bullet}\right)=2^{-\sup \left\{k \in \mathbb{N}_{0} \mid U_{k}\left(G^{\bullet}\right) \simeq U_{k}\left(H^{\bullet}\right)\right\}}
$$

with $U_{k}\left(G^{\bullet}\right) \simeq U_{k}\left(H^{\bullet}\right)$ denoting isomorphism of rooted graphs. This defines a pre-metric on the collection of all rooted locally finite connected graphs. Two such graphs have distance zero if and only if they are isomorphic. Hence this yields a metric on the collection of (representatives of) all unlabelled, connected, rooted, locally finite graphs. The resulting space $\mathbb{B}$ is Polish, meaning that standard probabilistic convergence concepts apply. For example, a random rooted graph $G^{\bullet} \in \mathbb{B}$ is the local weak limit of a sequence $\mathrm{G}_{n}^{\bullet}=\left(\mathrm{G}_{n}, v_{n}\right), n \in \mathbb{N}$ of random elements of this space if it is the weak limit with respect to this metric; that is, if

$$
\lim _{n \rightarrow \infty} \mathbb{E}\left[f\left(\mathrm{G}_{n}^{\bullet}\right)\right]=\mathbb{E}\left[f\left(\mathrm{G}^{\bullet}\right)\right]
$$

for any bounded continuous function $f: \mathbb{B} \rightarrow \mathbb{R}$. The neighbourhood projections $\left(U_{k}(\cdot)\right)_{k \geqslant 0}$ form a convergence determining family, meaning that $\mathrm{G}_{n}^{\bullet} \stackrel{d}{\longrightarrow} \mathrm{G}^{\bullet}$ is equivalent to

$$
\lim _{n \rightarrow \infty} \mathbb{P}\left(U_{k}\left(G_{n}^{\bullet}\right) \simeq G^{\bullet}\right)=\mathbb{P}\left(U_{k}\left(G^{\bullet}\right) \simeq G^{\bullet}\right)
$$

for any rooted graph $G^{\bullet}$ and all integers $k \geqslant 0$. If we use the conditional distribution of $v_{n}$ given that the graph $G_{n}$ is uniform on the vertex set $V\left(G_{n}\right)$, then the limit $G^{\bullet}$ is often also called the Benjamini-Schramm limit of the sequence $\left(\mathrm{G}_{n}\right)_{n}$.

\subsubsection{Local convergence of random unlabelled $k$-trees}

Our second main result is a local weak limit for $U_{n}$ that describes the asymptotic behaviour of the $r$-neighbourhoods $U_{r}\left(\mathrm{U}_{n}, v^{*}\right)$ of a uniform random vertex $v^{*}$ of the graph $\mathrm{U}_{n}$. We even obtain convergence in total variation of these neighbourhoods when $r=r_{n}$ depends on $n$ and satisfies $r_{n}=o(\sqrt{n})$. More precisely, we have the following. 
Theorem 2.3 The random rooted unlabelled $k$-tree $\left(\mathrm{U}_{n}, v_{n}\right)$ converges in the Benjamini-Schramm sense towards a random infinite rooted $k$-tree $\hat{U}$. Furthermore, for each sequence $r_{n}=o(\sqrt{n})$,

$$
d_{\mathrm{TV}}\left(U_{r_{n}}\left(\mathrm{U}_{n}, v_{n}\right), U_{r_{n}}(\hat{\mathrm{U}})\right) \rightarrow 0
$$

where $v_{n}$ denotes a uniformly selected vertex of $\mathrm{U}_{n}$.

In other words,

$$
\lim _{n \rightarrow \infty} \sup _{A}\left|\mathbb{P}\left(U_{r_{n}}\left(U_{n}, v_{n}\right) \in A\right)-\mathbb{P}\left(U_{r_{n}}(\hat{U}) \in A\right)\right|=0,
$$

with the index $A$ ranging over all countable collections of finite rooted graphs.

This strengthened form of convergence is best possible: Theorem 2.2 asserts that the diameter of the random unlabelled $k$-tree $\mathrm{U}_{n}$ has order $\sqrt{n}$. Since the diameter of $\hat{U}$ is almost surely infinite, the local convergence of $\mathrm{U}_{n}$ towards $\hat{U}$ fails for $r_{n}$-neighbourhoods if $n^{-1 / 2} r_{n}$ does not converge to zero.

We emphasize again that we treat random unlabelled $k$-trees. The second author [23] established a Benjamini-Schramm limit that describes the asymptotic behaviour of the vicinity of a typical vertex in random labelled $k$-trees.

\subsection{Asymptotic enumeration}

The cycle-pointing approach also allows us to recover the asymptotic expression

$$
u_{n} \sim \frac{\left(k \rho_{k}\right)^{-k}}{k^{2} k ! \sqrt{2 \pi}}\left(1+k \sum_{\ell \geqslant 2} \bar{B}_{1^{k}}^{\prime}\left(\rho_{k}^{\ell}\right) \rho_{k}^{\ell}\right)^{3 / 2} n^{-5 / 2} \rho_{k}^{-n}
$$

for the number $u_{n}$ of unlabelled $k$-trees with $n$ hedra that was proved by Drmota and the first author in [7, Theorem 3] via the dissymmetry equation. See Section 5.4 for details. Unlabelled graphs were recently studied using the cycle-pointing method in [24], but the results do not apply to $k$-trees for $k \geqslant 2$.

\section{The Gainer-Dewar-Gessel decomposition}

\subsection{Vertex colourings, hedron labellings and a bijection with $\boldsymbol{k}$-coding trees}

We recall some results and terminology from $[13,14]$. Any two hedra $h_{1}$ and $h_{2}$ in a $k$-tree that intersect at a front $f$ are termed adjacent. If this is the case, then a front $f_{1}$ of $h_{1}$ and a front $f_{2}$ of $h_{2}$ are called mirror with respect to $f$ if $f_{1} \cap f=f_{2} \cap f$.

A coloured hedron-labelled $k$-tree with $n$ hedra is a $k$-tree where the hedra are labelled by distinct integers from $[n]$ and the fronts are coloured with integers from $[k+1]$. We require that any two distinct fronts that belong to the same hedron must have distinct colours, and any two distinct fronts that are mirror with respect to some other front must have the same colour. This way, the $k+1$ fronts belonging to any single hedron are coloured with distinct integers from $[k+1]$. See Figure 2 for two examples where labels are denoted by boxed integers.

It is not hard to see that the colours of all fronts of any single hedron already determine the colours of all other fronts in the $k$-tree. However, the total number of front-colourings may vary according to the $k$-tree we consider.

We now introduce $k$-coding trees. A (coloured and labelled) $k$-coding tree is an unordered tree with a proper bipartition of its vertex set into white and black vertices. We require that each black vertex has precisely $k+1$ white neighbours. The $n$ black vertices are labelled with distinct integers from $[n]$, and to each white vertex we assign a colour from $[k+1]$, such that each black vertex has precisely one neighbour with colour $i$ for all $i \in[k+1]$. 

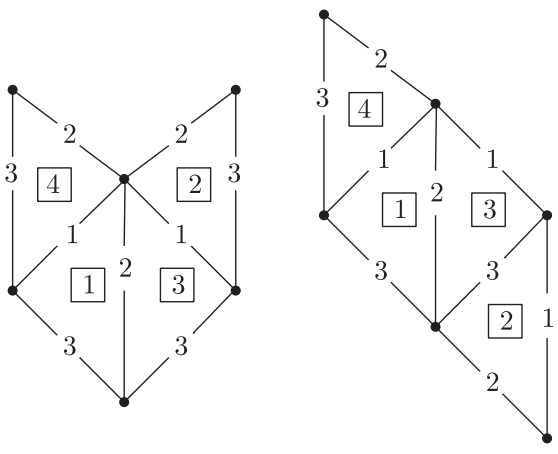

Figure 2. Two coloured hedron-labelled 2-trees with four hedra.

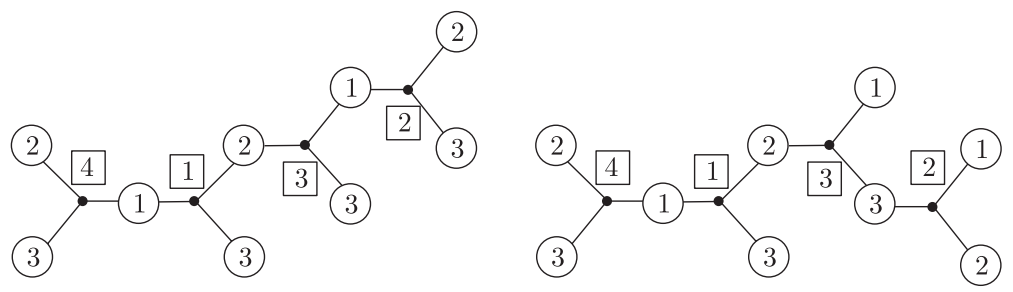

Figure 3. Two 2-coding trees that respectively correspond to the 2-trees in Figure 2.

There is a bijection $\phi$ between the set $\mathcal{K}_{n, k}$ of coloured hedron-labelled $k$-trees with $n$ hedra, and the set $\mathcal{T}_{n, k}$ of (coloured and labelled) coding trees with $n$ black vertices; see [14], which is analogous to the following [13, Theorem 3.4].

To construct a $k$-coding tree from a coloured hedron-labelled $k$-tree, we assign to each hedron a black vertex with the same label and to each front a white vertex with the same colour. We connect a white vertex with a black vertex by an edge if the front corresponding to the white vertex is a $k$-subset of the hedron corresponding to the black vertex.

For the inverse map, note that in order to construct a $k$-tree from a $k$-coding tree we require knowledge of the colouring. There are multiple ways to glue two front-coloured hedra together at a specified front colour, but only one way such that afterwards any pair of fronts that are mirror with respect to the resulting shared front have the same colour. For example, Figure 3 depicts two 2-coding trees that differ only in the colouring. Figure 2 shows the corresponding coloured hedron-labelled 2-trees. Note that the 2-trees in this example remain different even if we forget about their colours and labels.

For any integer $n \geqslant 0$ we let $\mathfrak{S}_{n}$ denote the symmetric group of degree $n$. The groups $\mathfrak{S}_{n}$ and $\mathfrak{S}_{k+1}$ both operate on the set $\mathcal{K}_{n, k}$ of coloured hedron-labelled $k$-trees, and the two actions commute. This induces an action of the group $\mathfrak{S}_{n}$ on the set $\mathcal{K}_{n, k} / \mathfrak{S}_{k+1}$ of orbits, which may be identified with $k$-trees on labelled hedra.

Any graph isomorphism between $k$-trees also induces a bijection between their sets of hedra. Thus, any two hedron-labelled $k$-trees are identical as unlabelled graphs if and only if one may be obtained from the other via relabelling of hedra. Thus the $\mathfrak{S}_{n}$-orbits of the induced action correspond precisely to the unlabelled $k$-trees with $n$ hedra.

Since the two actions on $\mathcal{K}_{n, k}$ commute, it follows that there is also a canonical correspondence between unlabelled $k$-trees and orbits of the group action of $\mathfrak{S}_{k+1}$ on the set $\mathcal{K}_{n, k} / \mathfrak{S}_{n}$. Elements of $\mathcal{K}_{n, k} / \mathfrak{S}_{n}$ correspond to $k$-trees that are unlabelled but coloured. Hence we refer to the $\mathfrak{S}_{k+1}$-orbits of this group action as colour-orbits of unlabelled $k$-trees.

As the bijection $\phi$ is compatible with the actions of both groups $\mathfrak{S}_{n}$ and $\mathfrak{S}_{k+1}$, this reduces the study of unlabelled $k$-trees to the study of colour-orbits of unlabelled $k$-coding trees, that is, orbits of the group action of $\mathfrak{S}_{k+1}$ on $\mathcal{T}_{n, k} / \mathfrak{S}_{n}$. 


\subsection{Burnside's lemma}

The enumeration of colour-orbits of unlabelled $k$-trees and $k$-coding trees is undertaken using Burnside's lemma, which we briefly recall in this section. Given a permutation $\sigma \in \mathfrak{S}_{m}$, its cycle type $\lambda=\left(1^{\lambda_{1}} 2^{\lambda_{2}} \cdots m^{\lambda_{m}}\right)$ is defined by letting $\lambda_{i}$ denote the number of cycles of length $i$ in the cycle decomposition of $\sigma$. Sometimes we simply write the parts of $\lambda$ in a weakly decreasing manner, that is,

$$
\lambda=(\underbrace{m, \ldots, m}_{\lambda_{m}}, \ldots, \underbrace{2, \ldots, 2}_{\lambda_{2}}, \underbrace{1, \ldots, 1}_{\lambda_{1}}) .
$$

We will also omit the parentheses when there is no risk of confusion. Since $m=\lambda_{1}+2 \lambda_{2}+\cdots+$ $m \lambda_{m}$, the cycle type $\lambda$ may be identified with a partition of $m$. That is, a permutation with $\lambda_{i}$ cycles of length $i$ for each $i$ corresponds to the partition in which the multiplicity of $i$ as a part is $\lambda_{i}$. We write $\lambda \vdash m$ to denote that $\lambda$ is a partition of $m$, and set

$$
z_{\lambda}=1^{\lambda_{1}} \lambda_{1} ! 2^{\lambda_{2}} \lambda_{2} ! \cdots m^{\lambda_{m}} \lambda_{m} !
$$

so that $m ! / z_{\lambda}$ is the number of permutations in $\mathfrak{S}_{m}$ with cycle type $\lambda$. Moreover, for any $d \geqslant 1$, we let $\lambda^{d}$ denote the cycle type of the $d$ th power of a fixed permutation with cycle type $\lambda$.

We let $A \subset \mathbb{R}[[z]]$ denote the subset of all formal power series whose coefficients are nonnegative. Suppose that we are given a non-empty set $S$ together with a weight-function $\omega: S \rightarrow$ $A$, such that the sum $\sum_{s \in S} \omega(s)$ is well-defined in $A$. That is, for any $n \geqslant 0$, the coefficients $\left(\left[z^{n}\right] \omega(s)\right)_{s \in S}$ form a summable family of non-negative real numbers. Suppose that we are additionally given a group action of the symmetric group $\mathfrak{S}_{m}$ on $S$ that preserves weights. Thus, all elements of a common orbit $O$ have the same $\omega$-weight, which we denote by $\omega(O)$ and call the weight of the orbit $O$. For each permutation $\sigma \in \mathfrak{S}_{m}$, we let $\operatorname{Fix}(\sigma)=\{s \in S \mid \sigma . s=s\}$ denote the set of fixed points of $\sigma$. The corresponding inventory $\operatorname{Fix}_{\lambda}:=\sum_{s \in \operatorname{Fix}(\sigma)} \omega(s)$ only depends on the cycle type $\lambda \vdash m$ of $\sigma$.

Lemma 3.1 (Burnside's lemma for the symmetric group). The sum of the weights of all $\mathfrak{S}_{m}$-orbits is given by

$$
\sum_{O \in S / \mathfrak{S}_{m}} \omega(O)=\sum_{\lambda \vdash m} \frac{\operatorname{Fix}_{\lambda}}{z_{\lambda}} .
$$

Suppose that for each type $\lambda \vdash m$ we fix some permutation $\sigma_{\lambda} \in \mathfrak{S}_{m}$ with type $\lambda$. Let

$$
Z_{m}=\sum_{O \in S / \mathfrak{S}_{m}} \omega(O)
$$

denote the sum of the weights of all orbits. The following probabilistic application of Burnside's lemma will turn out to be useful.

Lemma 3.2 Suppose that all $\omega$-weights are positive real numbers. We may sample a random type $\bar{\lambda} \vdash m$ with probability

$$
\mathbb{P}(\bar{\lambda}=\lambda)=z_{\lambda}^{-1} Z_{m}^{-1} \operatorname{Fix}_{\lambda}
$$

and then select an element $\bar{s}$ from $\operatorname{Fix}\left(\sigma_{\bar{\lambda}}\right)$ with probability proportional to its $\omega$-weight. Then the orbit $\bar{O}$ corresponding to $\bar{s}$ is distributed according to

$$
\mathbb{P}(\bar{O}=O)=\omega(O) Z_{m}^{-1} .
$$


Proof. Let $O \in S / \mathfrak{S}_{m}$ be an arbitrary orbit. Clearly the symmetric group $\mathfrak{S}_{m}$ also operates on $O$, and applying Burnside's lemma (Lemma 3.1) to this operation yields

$$
\omega(O)=\sum_{\lambda \vdash m} z_{\lambda}^{-1} \sum_{s \in \operatorname{Fix}\left(\sigma_{\lambda}\right) \cap O} \omega(s) .
$$

Hence

$$
\begin{aligned}
\mathbb{P}(\bar{O}=O) & =\sum_{\lambda \vdash m} \mathbb{P}(\bar{\lambda}=\lambda) \mathbb{P}\left(\bar{s} \in \operatorname{Fix}\left(\sigma_{\lambda}\right) \cap O \mid \bar{\lambda}=\lambda\right) \\
& =\sum_{\lambda \vdash m}\left(\frac{\operatorname{Fix}_{\lambda}}{Z_{m} z_{\lambda}}\right)\left(\sum_{s \in \operatorname{Fix}\left(\sigma_{\lambda}\right) \cap O} \frac{\omega(s)}{\operatorname{Fix}_{\lambda}}\right) \\
& =Z_{m}^{-1} \omega(O) .
\end{aligned}
$$

The operation of the group $\mathfrak{S}_{m}$ on the set $S$ induces an operation on the set $M(S)$ of all finite multi-sets of elements in $S$. The weight-function $\omega$ on $S$ extends in a natural way to $M(S)$ by defining the weight of a multi-set to be the product of the weights of its elements (with repetitions). For any $\sigma \in \mathfrak{S}_{m}$, we let $\operatorname{Fix}^{M(S)}(\sigma)$ denote the set of all $M \in M(S)$ satisfying $\sigma \cdot M=M$.

Lemma 3.3 ([14, Lemma 2]). For each $\sigma \in \mathfrak{S}_{m}$,

$$
\sum_{M \in \operatorname{Fix}^{M(S)}(\sigma)} \omega(M)=\exp \left(\sum_{i \geqslant 1} \frac{1}{i} \sum_{s \in \operatorname{Fix}^{S}\left(\sigma^{i}\right)} \omega(s)^{i}\right) .
$$

In [14, Lemma 2] a similar result was stated. However, instead of taking the power $\omega(s)^{i}$ on the right-hand side, the substitution operation $\omega(s)\left(z^{i}\right)$ was employed. (This makes no difference for the special cases in which this result is applied in [14], because there $\omega(s)$ is always some power of $z$ and the two operations coincide.)

Proof of Lemma 3.3. A multiset $M \in M(S)$ is fixed by $\sigma$ if and only if it is a multi-set union of orbits of $S$ under the action of the generated subgroup $\langle\sigma\rangle$. So let $\left(O_{j}\right)_{j \in J}$ denote the collection of these orbits. For each $j \in J$, we set $r_{j}=\left|O_{j}\right|$ and select a representative $s_{j} \in O_{j}$. Any $M \in M(S)$ may be written uniquely as a multi-set union of $\ell_{j} \geqslant 0$ copies of $O_{j}$ for all $j \in J$, with $\sum_{j \in J} \ell_{j}<\infty$. It follows that

$$
\sum_{M \in \operatorname{Fix}^{M(S)}(\sigma)} \omega(M)=\prod_{j \in J} \sum_{\ell_{j} \geqslant 0} \omega\left(s_{j}\right)^{\ell_{j} r_{j}}=\prod_{j \in J} \frac{1}{1-\omega\left(s_{j}\right)^{r_{j}}} .
$$

Here we have used the assumption that the family $(\omega(s))_{s \in S}$ is summable, which implies that all products with infinitely many factors $\neq 1$ in equation (3.3) vanish. That is, we really only sum up weights of finite multi-sets. Applying the logarithm operator to equation (3.3) yields

$$
\log \left(\sum_{M \in \mathrm{Fix}^{M(S)}(\sigma)} \omega(M)\right)=\sum_{j \in J} \sum_{\ell \geqslant 1} \frac{\omega\left(s_{j}\right)^{\ell r_{j}}}{\ell} .
$$

We now focus on the argument of the exponential operator on the right-hand side of equation (3.2). Clearly we may write

$$
\sum_{i=1}^{\infty} \frac{1}{i} \sum_{s \in \operatorname{Fix}^{S}\left(\sigma^{i}\right)} \omega(s)^{i}=\sum_{j \in J} \sum_{i \geqslant 1} \frac{1}{i} \sum_{s \in O_{j} \cap \mathrm{Fix}^{S}\left(\sigma^{i}\right)} \omega(s)^{i} .
$$


It holds that $O_{j} \subset \operatorname{Fix}^{S}\left(\sigma^{i}\right)$ if $i$ is a multiple of $r_{j}$, and $O_{j} \cap \operatorname{Fix}^{S}\left(\sigma^{i}\right)=\varnothing$ otherwise. Hence

$$
\sum_{j \in J} \sum_{i \geqslant 1} \frac{1}{i} \sum_{s \in O_{j} \cap \mathrm{Fix}^{S}\left(\sigma^{i}\right)} \omega(s)^{i}=\sum_{j \in J} \sum_{\ell \geqslant 1} \frac{\omega(s)^{\ell r_{j}}}{\ell} .
$$

Together with equation (3.4), this verifies equation (3.2).

\subsection{Generating functions}

We let $U(z)$ denote the generating series of unlabelled (uncoloured) $k$-trees indexed by their number of hedra. Equivalently, we may state that $U(z)$ is the generating series of colour-orbits of unlabelled (coloured) $k$-coding trees, indexed by their number of black vertices. The dissymmetry theorem proved by Gainer-Dewar and Gessel [14, Lemma 5, 6] expresses this function by the equation

$$
U(z)=B(z)+C(z)-E(z) .
$$

Here $B(z), C(z)$ and $E(z)$ denote the generating functions for colour-orbits of unlabelled (coloured) $k$-coding trees that are rooted at a black vertex, a white vertex and an edge, respectively. That is, the coefficient of $z^{n}$ in these series counts the number of $\mathfrak{S}_{k+1}$-orbits of unlabelled (coloured) $k$-coding trees $T$ of size $n$ (having $n$ black vertices) that are rooted at $v$, which is a black vertex, a white vertex or an edge.

Our goal in Section 5 is to provide a substraction-free alternative (equation (5.8)) to equation (3.5), based on the results of Gainer-Dewar and Gessel [13, Theorem 7] concerning $k$-coding trees rooted at a black or a white vertex. These classes may be decomposed by applying Lemma 3.3 and Burnside's lemma (Lemma 3.1) to recolouring operations on rooted, unlabelled and coloured objects. We briefly recall the arguments in [14], as we are going to use these decompositions (rather than just the resulting equations of generating functions) later on.

For any cycle type $\lambda \vdash k+1$, we fix a permutation $\pi_{\lambda} \in \mathfrak{S}_{k+1}$ having type $\lambda$ and let $B_{\lambda}(z)$ denote the generating function of coloured, unlabelled black-rooted $k$-coding trees that are invariant under $\pi_{\lambda}$. Furthermore, for any $i \geqslant 1$ we recall that $\lambda^{i}$ denote the cycle type of $\left(\pi_{\lambda}\right)^{i}$. This notion does not depend on the choice of $\pi_{\lambda}$. Burnside's lemma (Lemma 3.1) yields

$$
B(z)=\sum_{\lambda \vdash k+1} \frac{B_{\lambda}(z)}{z_{\lambda}} .
$$

Each colour-orbit of a $C$-object contains a coloured, unlabelled coding-tree where the white root vertex has colour $k+1$. Thus the colour-orbits of the action of $\mathfrak{S}_{k+1}$ on all white-rooted, coloured, unlabelled $k$-coding trees correspond precisely to the colour-orbits of the action of $\mathfrak{S}_{k}$ on coloured, unlabelled $k$-coding trees marked at a white vertex with colour $k+1$. Applying Burnside's lemma to this action of $\mathfrak{S}_{k}$ yields

$$
C(z)=\sum_{\mu \vdash k} \frac{C_{\mu}(z)}{z_{\mu}}
$$

with $C_{\mu}(z)$ denoting the generating series of all coloured unlabelled $k$-coding trees that are rooted at a white vertex with colour $k+1$ and that are invariant under recolouring by a fixed (but arbitrary) permutation $\sigma_{\mu} \in \mathfrak{S}_{k}$ with cycle type $\mu$.

We define the generating function $\bar{B}_{\mu}(z)$ in the same way as $C_{\mu}(z)$, but only count the $k$-coding trees where the white root with colour $k+1$ has precisely one black neighbour. This black neighbour may be interpreted as a black root vertex and $\bar{B}_{\mu}$-objects are termed black-rooted reduced $k$-coding trees. 
We may view a white-rooted, coloured, unlabelled $k$-coding tree whose root has colour $k+1$ as a multi-set of black-rooted reduced $k$-coding trees. Hence Lemma 3.3 applies, yielding

$$
C_{\mu}(z)=\exp \left(\sum_{i=1}^{\infty} \frac{\bar{B}_{\mu^{i}}\left(z^{i}\right)}{i}\right)
$$

If we delete the root of a $\bar{B}_{\mu}$-object, we are left with $k$ white-rooted unlabelled coloured $k$-coding trees $T_{1}, T_{2}, \ldots, T_{k}$ whose roots are coloured from 1 to $k$. For any cycle $c=\left(c_{1}, c_{2}, \ldots, c_{\ell}\right)$ of $\sigma_{\mu}$, the trees $T_{c_{1}}, T_{c_{2}}, \ldots, T_{c_{\ell}}$ belong to the same colour-orbit, and each is invariant under relabelling by $\sigma_{\mu}^{\ell}$. Setting $d=\min \left(c_{1}, c_{2}, \ldots, c_{\ell}\right)$, the result of switching the colour $d$ with the colour $k+1$ in the tree $T_{d}$ yields a reduced tree, that together with the cycle $c$ already contains all information on the trees $T_{c_{1}}, T_{c_{2}}, \ldots, T_{c_{\ell}}$. Hence the trees corresponding to $c$ are enumerated by $C_{\mu^{\ell}}\left(z^{\ell}\right)$, and the generating series for $\bar{B}_{\mu}$-objects is given by

$$
\bar{B}_{\mu}(z)=z \prod_{i \in \mu} C_{\mu^{i}}\left(z^{i}\right),
$$

with the index $i$ ranging over all parts of the type $\mu$. Similarly, we may argue that

$$
B_{\lambda}(z)=z \prod_{i \in \lambda} C_{\left(\lambda^{i}\right)^{-}}\left(z^{i}\right)
$$

with $\left(\lambda^{i}\right)^{-}$denoting the cycle type obtained by removing one part of length 1 from $\lambda^{i}$. In fact, by definition $C_{\lambda^{i}}\left(z^{i}\right)=C_{\left(\lambda^{i}\right)^{-}}\left(z^{i}\right)$ and $\lambda^{i}$ contains at least one part of length 1 . For example, when $k=2$, we have $\bar{B}_{2}(z)=z C_{1,1}\left(z^{2}\right)$ and $B_{2,1}(z)=z C_{1,1}\left(z^{2}\right) C_{2}(z)$.

\section{4. $k$-trees rooted at a front of distinguishable vertices}

We let $\rho_{k}$ denote the radius of convergence of the generating series $U(z)$ of unlabelled $k$-trees. Drmota and the first author [7] established the following result, which shows the dominating role of the cycle type $1^{k}$ in this context.

Lemma 4.1 ([7, Theorem 3]). The series $C_{1^{k}}(z)$ and $\bar{B}_{1^{k}}(z)$ have a dominant singularity of squareroot type at $z=\rho_{k}<1$ and it holds that $\bar{B}_{1^{k}}\left(\rho_{k}\right)=k^{-1}$. The series $C_{\mu}(z)$ and $\bar{B}_{\mu}(z)$ are analytic at $\rho_{k}$ if $\mu \neq 1^{k}$. The series $U(z)$ has a dominant singularity of type $\left(1-z / \rho_{k}\right)^{-3 / 2}$.

The class of labelled $k$-trees admits a recursive decomposition [8] that is based on $k$-trees rooted at a front of distinguishable vertices. Two such elements are considered isomorphic if there is a graph isomorphism that pointwise preserves the root-front. Hence the corresponding cycle-index sums do not count front-rooted unlabelled $k$-trees, but unlabelled $k$-trees that are rooted at a front of distinguishable vertices.

This relates to the present setting as follows. The $k$-trees counted by $\bar{B}_{1 k}(z)$ are unlabelled and coloured, with a root-front of colour $k+1$ that is contained in a unique hedron. The colours 1 to $k$ of the remaining fronts of this hedron uniquely determine the front-colouring of the entire $k$-tree, and may be interpreted as a labelling of the $k$ vertices of the root-front. That is, $\bar{B}_{1^{k}}(z)$ counts unlabelled uncoloured $k$-trees that are rooted at a front of $k$ distinguishable vertices that is contained in a unique hedron. The series $C_{1 k}(z)$ counts such objects without the restraint that the root-front belongs to a unique hedron. By (3.8) and (3.9) these series satisfy the equations

$$
\bar{B}_{1^{k}}(z)=z \exp \left(k \sum_{i=1}^{\infty} \frac{\bar{B}_{1^{k}}\left(z^{i}\right)}{i}\right) \quad \text { and } \quad C_{1^{k}}(z)=\exp \left(\sum_{i=1}^{\infty} \frac{\bar{B}_{1^{k}}\left(z^{i}\right)}{i}\right),
$$


which agree with the cycle-index sums associated to the decomposition of labelled $k$-trees in [8].

In [27] $k$-trees rooted at a front of distinguishable vertices were studied as special cases of unlabelled $\mathcal{R}$-enriched trees. Let $c_{k}$ be defined as in (2.8).

Lemma 4.2 ([27, Section 6.5]). Let $\mathrm{G}_{n}$ be either the uniform $n$-hedra $k$-tree from the class $\bar{B}_{1^{k}}$ or $C_{1^{k}}$. Let $\mu_{n}^{G}$ denote the uniform measure on the vertices of $\mathrm{G}_{n}$. Then the rescaled space $\left(\mathrm{G}_{n}, c_{k} n^{-1 / 2} d_{\mathrm{G}_{n}}, \mu_{n}^{\mathrm{G}}\right)$ converges in the Gromov-Hausdorff-Prokhorov sense towards the Brownian tree.

Furthermore, there are constants $C, c>0$ such that $\mathbb{P}\left(\mathrm{D}\left(\mathrm{G}_{n}\right) \geqslant x\right) \leqslant C \exp \left(-c x^{2} / n\right)$ for all $n$ and $x \geqslant 0$. Let $v_{n}$ be a vertex sampled according to $\mu_{n}^{\mathrm{G}}$. There is an infinite rooted random graph $\hat{G}$ such that for any sequence $r_{n}=o(\sqrt{n})$ the $r_{n}$-neighbourhood $U_{r_{n}}(\cdot)$ satisfies $d_{T V}\left(U_{r_{n}}\left(\mathrm{G}_{n}, v_{n}\right), U_{r_{n}}(\hat{\mathrm{G}})\right) \rightarrow 0$.

Here the limit graph $\hat{G}$ does not depend on whether we consider random elements of the class $\bar{B}_{1^{k}}$ or of the class $C_{1^{k}}$. Note that the Gromov-Hausdorff convergence of $\mathrm{G}_{n}$ to the Brownian tree was established in [27], but it is not hard to see that the arguments may be extended to obtain the Gromov-Hausdorff-Prokhorov convergence. The scaling constant $c_{k}$ is explicit in [27, first display after equation (7.29)] and may be seen to be identical to the expression in (2.8) by straightforward calculations.

\section{A substraction-free decomposition}

\subsection{Cycle-pointing}

Let $m \geqslant 0$ be an integer. Recall that any permutation $\sigma \in \mathfrak{S}_{m}$ may be decomposed in a unique way into a product of disjoint cycles. The cycles correspond to the orbits of the action of the generated subgroup $\langle\sigma\rangle \subset \mathfrak{S}_{m}$ on the set of integers $[m]$. Here we count fixed points as 1-cycles.

Suppose that the symmetric group $\mathfrak{S}_{m}$ acts on a set $S$. We may consider the cycle-pointed set $S^{\circ}$ of all pairs $(s, c)$ of any element $s \in S$ together with a marked cycle $c$, for which at least one permutation $\sigma \in \mathfrak{S}_{m}$ satisfies $\sigma . s=s$ and $\sigma$ has $c$ as one of its cycles. Naturally, the group $\mathfrak{S}_{m}$ acts on $S^{\circ}$ via $v .(s, c)=\left(\nu . s, v c v^{-1}\right)$ for all $v \in \mathfrak{S}_{m}$ and $(s, c) \in S^{\circ}$.

There is a well-defined map $S^{\circ} / \mathfrak{S}_{m} \rightarrow S / \mathfrak{S}_{m}$ that sends the orbit of an element $(s, c) \in S^{\circ}$ to the orbit of $s$. By [5, Theorem 15], the pre-image of any orbit in $S / \mathfrak{S}_{m}$ has precisely $m$ elements. This completely reduces the task of counting $S / \mathfrak{S}_{m}$ to the task of counting $S^{\circ} / \mathfrak{S}_{m}$. The latter may be easier, as the marked cycle provides a point of reference.

Recall that the groups $\mathfrak{S}_{n}$ and $\mathfrak{S}_{k+1}$ operate on the class $\mathcal{K}_{n, k}$ of coloured hedron-labelled $k$ trees, and that the two operations commute. We would like to study unlabelled, uncoloured $k$-trees that correspond bijectively to the elements of the collection $\left(\mathcal{K}_{n, k} / \mathfrak{S}_{n}\right) / \mathfrak{S}_{k+1}$ of colour-orbits of unlabelled, coloured $k$-trees.

We have to take great care when trying to apply the cycle-pointing method to this setting, as there are luring pitfalls. For example, we could apply the cycle-pointing operation to the operation of $\mathfrak{S}_{n}$ on the set $\mathcal{K}_{n, k}$, resulting in a set $\mathcal{K}_{n, k}^{O_{n}}$. The orbits from $\mathcal{K}_{n, k}^{o_{n}} / \mathfrak{S}_{n}$ are in an $n$ to 1 correspondence to the unlabelled coloured $k$-trees from $\mathcal{K}_{n, k} / \mathfrak{S}_{n}$, but this relation breaks when passing to the colour-orbits. That is, in general the orbits from $\left(\mathcal{K}_{n, k}^{0_{n}} / \mathfrak{S}_{n}\right) / \mathfrak{S}_{k+1}$ no longer satisfy an $n$ to 1 correspondence to the unlabelled, uncoloured $k$-trees from $\left(\mathcal{K}_{n, k} / \mathfrak{S}_{n}\right) / \mathfrak{S}_{k+1}$. A counter-example where this relation fails is already given for the special case $n=5$ and $k=1$. See Figure 4 .

Let $\mathcal{U}_{n}$ denote the set of all unlabelled (uncoloured) $k$-trees with $n$ hedra. We are going to consider the action of the symmetric group $\mathfrak{S}_{n}$ on the set $\mathcal{K}_{n}$ of $n$-hedron $k$-trees with hedra labelled from 1 to $n$. Clearly there is a bijection from $\mathcal{K}_{n} / \mathfrak{S}_{n}$ to $\mathcal{U}_{n}$, and consequently an $n$ to 1 correspondence from $\mathcal{V}_{n}:=\mathcal{K}_{n}^{\circ} / \mathfrak{S}_{n}$ to $\mathcal{U}_{n}$. See Figure 5. 
(a)
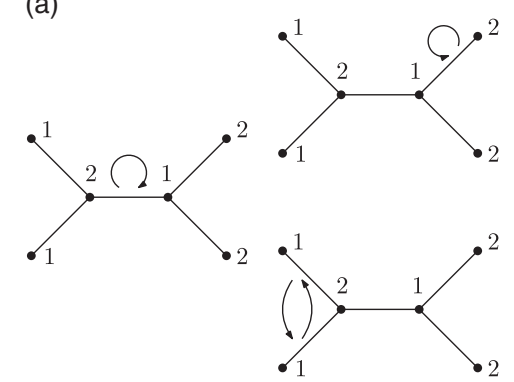
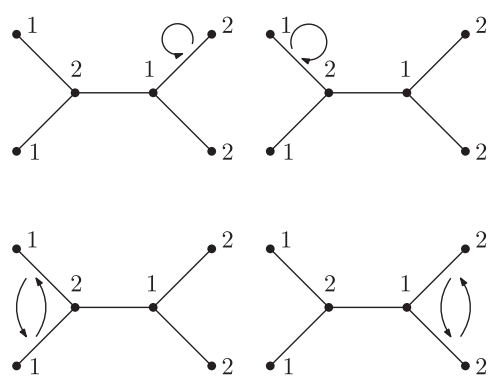

(b)

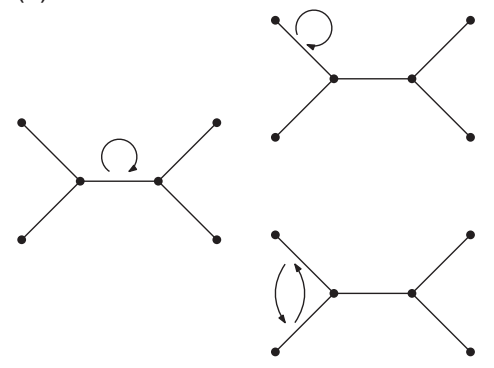

Figure 4. (a) All possible ways to first colour and then cycle-point a fixed unlabelled, uncoloured 1-tree with $n=5$ hedra (edges). (b) The three corresponding colour-orbits. This demonstrates that if we colour before applying the cycle-pointing operation, we break the $n$ to 1 relation between pointed and non-pointed objects.
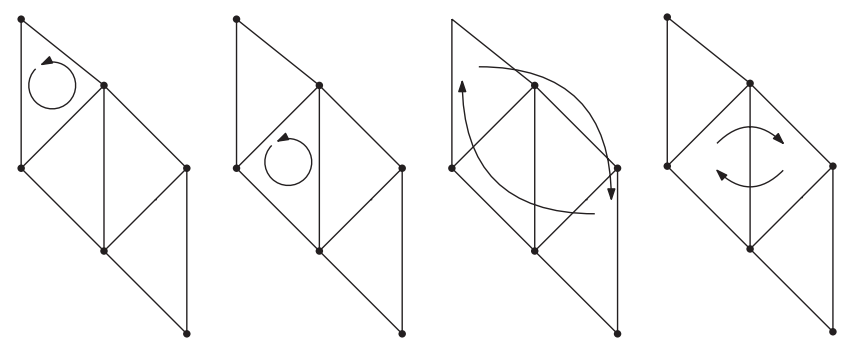

Figure 5. The four possible cycle-pointings of a 2-tree with four hedra.

We are going to partition the set $\mathcal{V}_{n}$ into three classes. We define the first class $\mathcal{V}_{n}^{(1)} \subset \mathcal{V}_{n}$ to be the subset of all unlabelled $k$-trees that are cycle-pointed at a single hedron. Hence the complement $\mathcal{V}_{n} \backslash \mathcal{V}_{n}^{(1)}$ consists of all unlabelled cycle-pointed $k$-trees whose marked cycle has length at least 2. Elements of the complement have a centre of symmetry or cycle-centre that may be either a hedron (as in Figure 6) or a front (as in Figure 7). The idea is that there is a common centre of paths that join consecutive atoms of the cycle in an associated $k$-coding tree.

Let us make this precise. Let $V \in \mathcal{V}_{n} \backslash \mathcal{V}_{n}^{(1)}$ be an unlabelled (uncoloured) cycle-pointed $k$-tree $V \in \mathcal{V}_{n}$ whose marked cycle has length $\ell \geqslant 2$. We may select a labelled version $(K, c) \in \mathcal{K}_{n}^{\circ}$ of $V$, and then a front-coloured (labelled) version $\left(K^{\mathrm{col}}, c\right) \in \mathcal{K}_{n, k}^{\circ}$ of $(K, c)$. Note that $c$ need not be a cycle of an automorphism of $K$ that preserves the colours; see Figure 7 for an example.

The labelled, coloured $k$-tree $K^{\text {col }} \in \mathcal{K}_{n, k}$ corresponds to a labelled, coloured $k$-coding tree $T \in \mathcal{T}_{n, k}$. We may form the tree $T^{\text {uncol }}$ by removing the colour-indicators from the white vertices. The pair $\left(T^{\mathrm{uncol}}, c\right)$ is a cycle-pointed tree (with labels on vertices instead of hedra) that no longer contains all information about the $k$-tree $V$. (We would require knowledge of the colours to reconstruct $V$.) We may consider the $\ell$ paths that join consecutive atoms of the marked cycle in $T^{\text {uncol }}$. Each of these paths has odd length since its ends are black vertices. It follows by a general principle for cycle-pointed trees [5, Proposition 24] that all connecting paths share a common centre, which may be either a black or a white vertex. We say that the corresponding hedron or front in $V$ is the cycle-centre (or centre of symmetry) of $V$. This notion does not depend on the choices of colours and labels in the intermediate steps.

Hence we may split $\mathcal{V}_{n}$ into a disjoint union

$$
\mathcal{V}_{n}=\mathcal{V}_{n}^{(1)} \sqcup \mathcal{V}_{n}^{(2)} \sqcup \mathcal{V}_{n}^{(3)} .
$$

The first part corresponds to unlabelled $k$-trees with a marked hedron. The second part corresponds to unlabelled $k$-trees with a marked cycle of length at least 2 , such that the cycle-centre is 
(a)

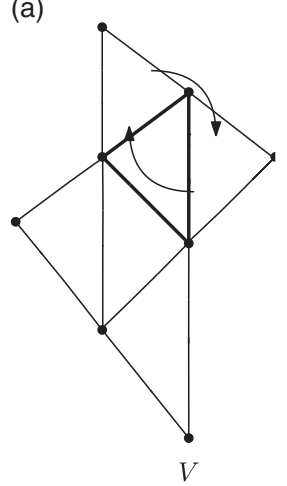

(b)

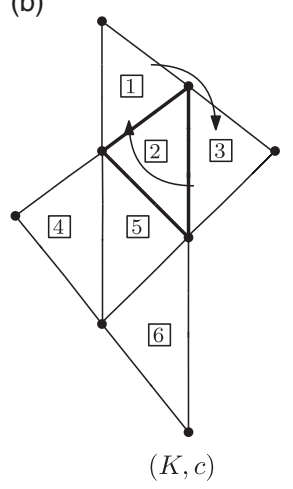

(c)

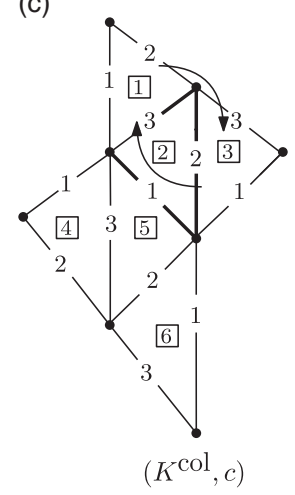

(d)

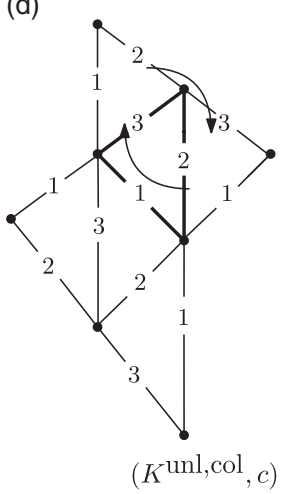

Figure 6. (a) $V$ is a cycle-pointed unlabelled uncoloured 2-tree from the set $\mathcal{V}_{6}^{(2)}$. The cycle-centre (a hedron) is drawn in bold. $(b, c)(K, c)$ denotes one of the cycle-pointed hedron-labelled (uncoloured) versions of $V$, and $\left(K^{\mathrm{col}}, c\right)$ is one of the front-coloured versions of $(K, c)$. (d) $\left(K^{\mathrm{unl}, \mathrm{col}}, c\right)$ is obtained by dropping the labels of $\left(K^{\mathrm{col}}, c\right)$.

a hedron, and the third part to the case where the cycle-centre is a front. We let $V(z)$, and $V^{(i)}(z)$, $i=1,2,3$ denote the corresponding generating series. That is,

$$
V^{(i)}(z)=\sum_{n \geqslant 1}\left|\mathcal{V}_{n}^{(i)}\right| z^{n} \quad \text { and } \quad V(z)=\sum_{n \geqslant 1}\left|\mathcal{V}_{n}\right| z^{n}
$$

\subsection{Structural analysis of the summands}

The generating series and bijective arguments of Section 3.3 may be interpreted in terms of $k$ coding trees (rooted for example at black or white vertices) and in terms of $k$-trees (rooted at a hedron or a front). In order to avoid confusion, we are going to interpret everything in terms of $k$ trees from here on. In particular, we regard $B(z)$ as the generating series of unlabelled, uncoloured $k$-trees rooted at a hedron, and $C(z)$ as the generating series of unlabelled, uncoloured $k$-trees that are rooted at a front. A front-colouring of a $k$-tree will always be subject to the restraints stated in Section 3.1, that is, the fronts of any hedron are coloured from 1 to $k+1$ and the fronts that are mirror to each other receive the same colour.

\subsubsection{Hedron-rooted $k$-trees}

It is clear that $\mathcal{V}^{(1)}$-objects are exactly unlabelled uncoloured hedron-rooted $k$-trees, that is,

$$
V^{(1)}(z)=B(z)
$$

\subsubsection{Cycle-pointed $k$-trees with a hedron cycle-centre}

In this subsection we show that there are only very few cycle-pointed $k$-trees with a hedron cyclecentre.

Lemma 5.1 There are constants $C, c>0$ that do not depend on $n$ such that

$$
\frac{\left|\mathcal{V}_{n}^{(2)}\right|}{\left|\mathcal{V}_{n}\right|} \leqslant C \exp (-c n)
$$

Proof. For this it suffices to show that the radius of convergence of the generating series $V^{(2)}(z)$ is strictly larger than the radius of convergence $\rho_{k}$ of the generating series $V(z)$. Indeed, if this is the 
case, then there is an $\epsilon>0$ such that $V^{(2)}\left(\rho_{k}+\epsilon\right)<\infty$ and hence $\left|\mathcal{V}_{n}^{(2)}\right|\left(\rho_{k}+\epsilon\right)^{n} \rightarrow 0$. As

$$
\left|\mathcal{V}_{n}\right|=n\left[z^{n}\right] U(z) \sim a_{k} n^{-3 / 2} \rho_{k}^{-n}
$$

for some fixed $a_{k}>0$ by Lemma 4.1 (or equation (5.9) below), we know that $\left|\mathcal{V}_{n}\right|\left(\rho_{k}+\varepsilon / 2\right)^{n} \rightarrow$ $\infty$. So

$$
\frac{\left|\mathcal{V}_{n}^{(2)}\right|}{\left|\mathcal{V}_{n}\right|}=o(1)\left(\frac{\rho_{k}+\varepsilon / 2}{\rho_{k}+\varepsilon}\right)^{n}
$$

tends exponentially fast to zero as $n$ becomes large, as desired.

It remains to verify that $V^{(2)}(z)$ is analytic at $z=\rho_{k}$. Let $V \in \mathcal{V}_{n}^{(2)}$ be a cycle-pointed unlabelled uncoloured $k$-tree whose cycle-centre is a hedron. Then there is a hedron-labelled, uncoloured $k$-tree $K$ with an automorphism $\sigma$ and a marked cycle $c$ of $\sigma$ such that $(K, c)$ looks like $V$, up to relabelling. See Figure 6 for a corresponding example in this proof.

We may view $K$ as rooted at the cycle-centre hedron. Hence $K$ consists of a root hedron whose fronts are identified with the root-fronts of $k+1$ front-rooted $k$-trees $C_{1}, \ldots, C_{k+1}$. If $\sigma$ sends the label of a hedron contained in $C_{i}$ to the label of a hedron contained in $C_{j}$, then it already holds that the restriction of $\sigma$ to the label set of $C_{i}$ is an isomorphism from $C_{i}$ to $C_{j}$. As the cycle centre is a hedron, it follows that there are branches $C_{i_{1}}, \ldots, C_{i_{\ell}}$ (each having at least 1 hedron) with $\ell \geqslant 2$ such that $\sigma$ cyclically permutes the label sets of the branches. That is, $\sigma$ induces an isomorphism from $C_{i_{j}}$ to $C_{i_{j+1}}$ if $1 \leqslant j<\ell$ and to $C_{i_{1}}$ if $j=\ell$.

Let $K^{\mathrm{col}}$ denote any fixed front-coloured version of $K$ (such that fronts of any hedron are coloured from 1 to $k+1$ and fronts that are mirror to each other receive the same colour). The automorphism $\sigma$ is not required to respect the colouring in the sense that $\sigma \cdot K=K$, but we know that when we relabel the hedra of $K^{\mathrm{col}}$ according to $\sigma$, then the result $\sigma \cdot K^{\mathrm{col}}$ must be some coloured version of $K$. Hence there is a bijection $\tau \in \mathfrak{S}_{k+1}$ such that

$$
\sigma \cdot K^{\mathrm{col}}=\tau \cdot K^{\mathrm{col}} \text {. }
$$

That is, the relabelled version $\sigma . K^{\mathrm{col}}$ equals the recoloured version $\tau \cdot K^{\mathrm{col}}$. See Figure 6 for an example of a cycle-pointed 2-tree $\left(K^{\mathrm{col}}, c\right)$ with $c=(13)$, where $\sigma \cdot K^{\mathrm{col}}=\tau \cdot K^{\mathrm{col}}$ holds for $\tau=(1)(23)$ and $\sigma=(13)(2)(46)(5)$.

Let $A \in[n]$ be the label of the cycle-centre hedron in $K^{\text {col }}$ and let $B \in[n]$ be the label of some hedron of $C_{i_{j}}$ that is next to the cycle-centre hedron. Then $\tau$ must map the colour $a_{i_{j}} \in[k+1]$ of the unique front contained in the hedra (corresponding to) $A$ and $B$ to the colour of the unique front contained in the hedra (corresponding to) $\sigma(A)=A$ and $\sigma(B)$. Thus $\left(a_{i_{1}}, \ldots, a_{i_{\ell}}\right)$ is one of the disjoint cyclic factors of the permutation $\tau$. As $\ell \geqslant 2$, this implies that $\tau$ does not have cycle type $1^{k+1}$.

Let $K^{\text {unl,col }}$ denote the result of dropping the labels of $K^{\text {col }}$ but retaining the colours. We know that $\tau \cdot K^{\mathrm{col}}=\sigma \cdot K^{\mathrm{col}}$ is a relabelled version of $K^{\mathrm{col}}$, so $K^{\mathrm{unl}, \mathrm{col}}$ is invariant under recolouring according to the permutation $\tau$. Thus $V$ is formed by dropping the colours of $K^{\text {unl,col }}$ and cyclepointing it in one of the at most $n$ ways such that the cycle centre is the root hedron. This shows that

$$
\left|\mathcal{V}_{n}^{(2)}\right| \leqslant n\left[z^{n}\right] \sum_{\substack{\lambda \vdash k+1 \\ \lambda \neq 1^{k+1}}} B_{\lambda}(z)=\left[z^{n-1}\right] \sum_{\substack{\lambda \vdash k+1 \\ \lambda \neq 1^{k+1}}} B_{\lambda}^{\prime}(z) .
$$

By Lemma 4.1 and equation (3.10) it follows that the series $B_{\lambda}^{\prime}(z)$ has radius of convergence strictly larger than $\rho_{k}$ for all $\lambda \neq 1^{k+1}$. This concludes the proof. 
(a)

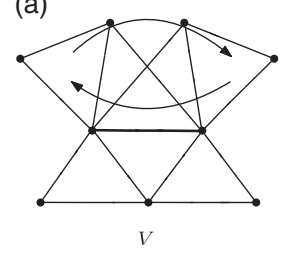

(b)

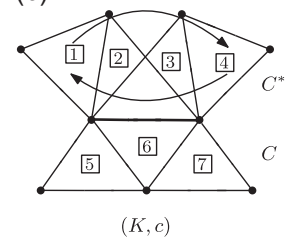

(c)

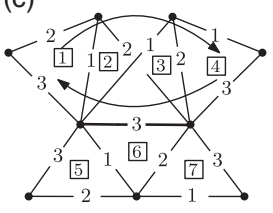

$\left(K^{\mathrm{col},}, c\right)$ (d)

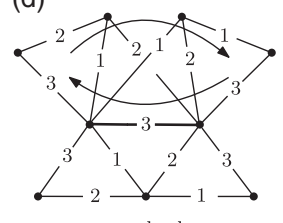

$\left(K^{\mathrm{unl}, \mathrm{col}}, c\right)$

Figure 7. (a) A cycle-pointed unlabelled 2-tree $V \in \mathcal{V}_{7}^{(3), \text { sym }}$. The cycle-centre (a front) is drawn in bold. (b) $(K, c)$ denotes a hedron-labelled version of $V$, and $C^{*}$ and $C$ denote the marked and unmarked part of $(K, C)$. (c,d) $K^{\text {col }}$ is a front-coloured version of $K$ and $K^{\mathrm{unl}, \mathrm{col}}$ is obtained by dropping the hedron-labels of $K^{\mathrm{col}}$.

\subsection{Cycle-pointed $k$-trees with a front cycle-centre}

Treating this case will require us to split it up into two subcases (see (5.4)).

Let $V \in \mathcal{V}_{n}^{(3)}$ be a cycle-pointed unlabelled uncoloured $k$-tree whose cycle-centre is a front. Then there is a hedron-labelled uncoloured $k$-tree $K$ together with an automorphism $\sigma$ and a marked cycle $c$ of $\sigma$ such that $V$ is the unlabelled version of $(K, c)$.

We consider $K$ as rooted at the cycle-centre front. Hence $K$ consists of a set of front-rooted hedron-labelled uncoloured $k$-trees where the root-front is contained in a unique hedron. We may decompose $K$ into branches that contain hedra of the marked cycle $c$ and branches that do not. Thus $K$ actually consists of two front-rooted components $C^{*}$ and $C$ that are glued together at their root-fronts, with $C^{*}$ the subgraph induced by all the branches containing hedra of the cycle $c$ and $C$ the subgraph induced by the branches that do not. We call $C^{*}$ the marked part and $C$ the unmarked part of $K$. See Figure 7 for a corresponding example.

If the automorphism $\sigma$ sends the label of a hedron contained in a branch $C_{1}$ of $K$ to a label of a hedron contained in another branch $C_{2}$, then the restriction of $\sigma$ to the label set of $C_{1}$ is an isomorphism from $C_{1}$ to $C_{2}$. In particular, $\sigma$ can be restricted to an automorphism of $C^{*}$ and consequently also to an automorphism of $C$.

Let us fix a version $K^{\mathrm{col}}$ of $K$ that is properly front-coloured such that fronts of any hedron are coloured from 1 to $k+1$ and fronts that are mirror to each other receive the same colour. We additionally require that the root-front has colour $k+1$. We know that the result of relabelling $K^{\mathrm{col}}$ according to $\sigma$ is a coloured version of $K$, where the root-front still has colour $k+1$. Consequently, there is a bijection $\pi \in \mathfrak{S}_{k}$ such that $\sigma \cdot K^{\mathrm{col}}=\pi \cdot K^{\mathrm{col}}$, that is, the relabelled version $\sigma \cdot K^{\mathrm{col}}$ equals the recoloured version $\pi \cdot K^{\mathrm{col}}$. Figure 7 illustrates an example where $c=(14)$ and $\sigma \cdot K^{\mathrm{col}}=\pi \cdot K^{\mathrm{col}}$ holds for $\pi=(12)(3)$ and $\sigma=(14)(23)(57)(6)$.

Let $\mathcal{V}_{n}^{(3), \text { sym }} \subset \mathcal{V}_{n}^{(3)}$ be the subset of all cycle-pointed $k$-trees where $\sigma$ and $\pi$ cannot be chosen in such a way that $\pi$ is the identity permutation. (This does not depend on the choice of $K^{\mathrm{col}}$.) See Figure 8(b) for an example of an element of $\mathcal{V}_{7}^{(3), \text { sym }}$. We set $\mathcal{V}_{n}^{(3), \text { dec }}:=\mathcal{V}_{n}^{(3)} \backslash \mathcal{V}_{n}^{(3) \text {,sym }}$, so that

$$
V^{(3)}(z)=V^{(3), \operatorname{sym}}(z)+V^{(3), \operatorname{dec}}(z)
$$

with

$$
V^{(3), \text { sym }}(z)=\sum_{n \geqslant 1}\left|\mathcal{V}_{n}^{(3), \text { sym }}\right| z^{n} \quad \text { and } \quad V^{(3), \operatorname{dec}}(z)=\sum_{n \geqslant 1}\left|\mathcal{V}_{n}^{(3), \operatorname{dec}}\right| z^{n}
$$

Our first observation tells us that $\mathcal{V}_{n}^{(3), \text { sym }}$ contains very few elements.

Lemma 5.2 There are constants $C, c>0$ that do not depend on $n$ such that

$$
\frac{\left|\mathcal{V}_{n}^{(3), \mathrm{sym}}\right|}{\left|\mathcal{V}_{n}\right|} \leqslant C \exp (-c n)
$$


(a)

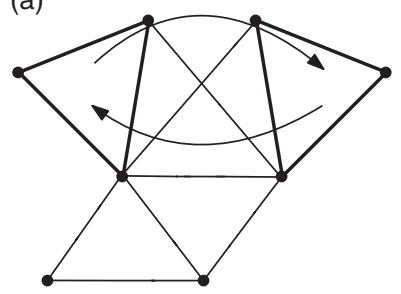

(b)

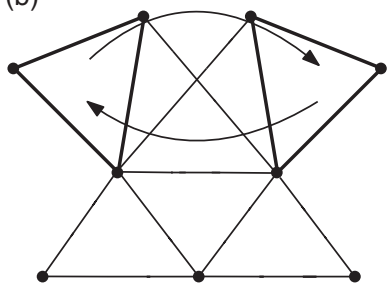

(c)

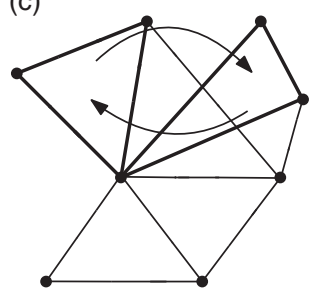

Figure 8. (a) The 2-tree is not cycle-pointed, since no automorphism has the marked cycle as one of its disjoint cyclic factors. (b,c) The other two are cycle-pointed with a front as cycle-centre: (b) belongs to the class $\mathcal{V}_{7}^{(3), \text { sym }}$, while (c) belongs to the class $\mathcal{V}_{6}^{(3), d e c}$.

Proof. Let $K^{\text {unl,col }}$ denote the unlabelled and coloured $k$-tree obtained by dropping the labels but retaining the colours of $K^{\mathrm{col}}$. As $\sigma \cdot K^{\mathrm{col}}=\pi \cdot K^{\mathrm{col}}$, it follows that $K^{\mathrm{unl} \text {,col }}$ is invariant under recolouring according to the permutation $\pi$. Since any unlabelled $k$-tree with $n$ hedra has at most $n$ unlabelled cycle-pointed versions where the cycle-centre is a front, it follows that

$$
\left|\mathcal{V}_{n}^{(3), \text { sym }}\right| \leqslant n\left[z^{n}\right] \sum_{\substack{\mu \vdash k \\ \mu \neq 1^{k}}} C_{\mu}(z)=\left[z^{n-1}\right] \sum_{\substack{\mu \vdash k \\ \mu \neq 1^{k}}} C_{\mu}^{\prime}(z) .
$$

By Lemma 4.1 we know that for each $\mu \vdash k$ with $\mu \neq 1^{k}$ the series $C_{\mu}^{\prime}(z)$ has radius of convergence strictly larger than $\rho_{k}$. This implies that the generating series $\sum_{n \geqslant 1}\left|\mathcal{V}_{n}^{(3) \text {,sym }}\right| z^{n}$ has radius of convergence strictly larger than $\rho_{k}$. Inequality (5.5) now follows analogously to (5.3) in the proof of Lemma 5.1.

Now we turn to the complement set $\mathcal{V}_{n}^{(3), \text { dec }}$. For $V \in \mathcal{V}_{n}^{(3) \text {,dec }}$, we may assume that the automorphism $\sigma$ got chosen in a way that preserves the colouring of $K^{\mathrm{col}}$ as well, that is, $\sigma \cdot K^{\mathrm{col}}=K^{\mathrm{col}}$. We argued above that the automorphism $\sigma$ restricts to an automorphism of the marked part $C^{*}$ (and to an automorphism of the unmarked part $C$ ). Consequently, at least one (and hence all) colourings of the marked part admit a colour-preserving automorphism having the marked cycle $c$ as one of its disjoint cyclic factors.

This is a key observation: arbitrary elements of $\mathcal{V}_{n}^{(3)}$ may have a marked part whose marked cycle may only be extended to automorphisms involving some form of rotation of the root hedron (i.e. they are not colour preserving) such as the $k$-tree of Figure $8(\mathrm{~b})$. This imposes symmetry constraints (i.e. invariance under non-trivial recolouring) on the unmarked part. For this reason we could show in (5.6) that there are far fewer elements in $\mathcal{V}_{n}^{(3) \text { sym }}$ than in $\mathcal{V}_{n}^{(3)}$. For elements of $\mathcal{V}_{n}^{(3), \text { dec }}$ there are no such symmetry constraints. If the marked cycle may be extended to an automorphism of the marked part $C^{*}$ that preserves a front-colouring, then $C$ may be equal to any front-rooted unlabelled $k$-tree such that the total number of hedra of $C$ and $C^{*}$ sum up to $n$. (The abbreviation 'dec' for 'decoupled' intends to indicate this.) In fact, we may always choose $\sigma$ in such a way that it pointwise fixes all hedra of $C$. Note that, given a marked and an unmarked part, there is in general no canonical way to glue them together at the root-front. We will get to this in a moment.

Let us first examine the constraints on the marked part. Since $\sigma$ preserves the colouring, this means that if we distinguish the vertices of the root-front of $C^{*}$ by ordering them linearly, then $C^{*}$ consists of identical branches glued together in the unique way according to the order on the rootfront. See for example the cycle-pointed 2-tree $(K, c)$ in Figure 9, where the branch containing hedra 1 and 2 is identical to the branch containing 3 and 4 , while the branch containing 5 and 6 can be any front-rooted hedron-labelled 2 -trees. 
(a)

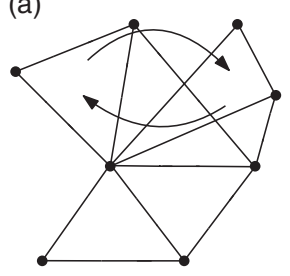

V (b)

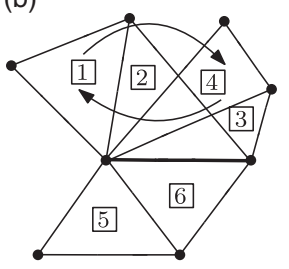

$(K, c)$ (c)

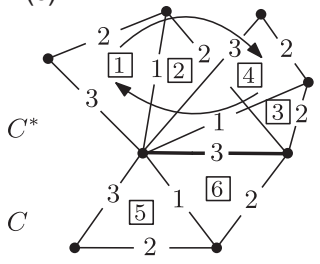

$\left(K^{\mathrm{col}}, c\right)$ (d)

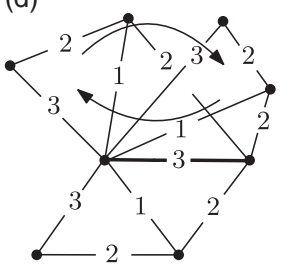

$\left(K^{\mathrm{unl}, \mathrm{col}}, c\right)$

Figure 9. (a) A cycle-pointed unlabelled 2-tree $V \in \mathcal{V}_{6}^{(3), d e c}$, (b) a cycle-pointed hedron-labelled version $K$ of $V$, (c,d) a front-coloured version $K^{\text {col }}$ of $K$ and $K^{\text {unl,col }}$ after dropping the labels of $K^{\mathrm{col}}$, where the cycle-centre (a front) is drawn in bold.

(b)

(a)

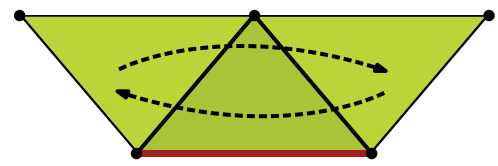

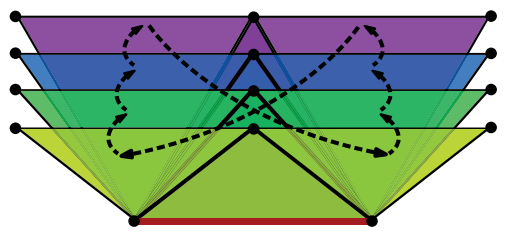

Figure 10. Constructing a marked part with a cycle of length 8 out of four copies of a single marked branch with a cycle of length 2.

The automorphism $\sigma$ cyclically permutes the label sets of the branches of $C^{*}$. Let $C_{1}, \ldots, C_{\ell}$, $\ell \geqslant 2$ denote the branches of $C^{*}$ such that $C_{i}$ is sent to $C_{i+1}$ by $\sigma$ if $i<\ell$, and to $C_{1}$ if $i=\ell$. The disjoint cyclic factor of $\sigma$ that corresponds to the marked cycle must be of the form

$$
\left(a_{1,1}, \ldots, a_{1, \ell}, a_{2,1}, \ldots, a_{2, \ell}, \ldots, a_{r, 1}, \ldots, a_{r, \ell}\right)
$$

for some $r \geqslant 1$ such that for each $1 \leqslant j \leqslant \ell$ the labels $a_{1, j}, a_{2, j}, \ldots, a_{r, j}$ correspond to distinct hedra of the branch $C_{j}$. Note that the restriction of the power $\sigma^{\ell}$ to the label set of $C_{j}$ is an automorphism of $C_{j}$, and $\left(a_{j, 1}, a_{j, 2}, \ldots, a_{j, \ell}\right)$ is one of its disjoint cyclic factors. Hence, up to hedron labels, $C^{*}$ is completely determined by the number $\ell \geqslant 2$ of branches together with a single cycle-pointed branch $\left(C_{j},\left(a_{1, j}, a_{2, j}, \ldots, a_{r, j}\right)\right)$. See Figure 10 for an illustration of how to construct a marked part in this canonical way.

Note that not every marked part constructed in this way is admissible. By admissible we mean that the marked part admits a front-colour preserving automorphism with the marked cycle as a factor. For instance, Figure 10(b) is not admissible.

Let $\bar{B}(z)$ be the generating series so that $\left[z^{n}\right] \bar{B}(z)$ counts the number of front-rooted unlabelled uncoloured $k$-trees with $n$ hedra where the root-front is contained in a unique hedron. Let $\bar{B}^{\circ \mathrm{w}}(z)$ count unlabelled uncoloured cycle-pointed branches that admit an automorphism that has the marked cycle as one of its disjoint factors and preserves a given (and hence all) front-colourings. (The ' $w$ ' indicates that they are 'well' pointed.) The generating series $M(z)$ of the class $\mathcal{M}$ of marked parts that are admissible for elements of the class $\mathcal{V}^{(3) \text {,dec }}=\bigcup_{n \geqslant 1} \mathcal{V}_{n}^{(3) \text {,dec }}$ is consequently given by

$$
M(z)=\sum_{\ell \geqslant 2} \bar{B}^{\circ}{ }_{\mathrm{w}}\left(z^{\ell}\right),
$$

with the variable $z$ indexing the number of hedra.

As mentioned before, there may be various ways to glue an unlabelled uncoloured marked part and a front-rooted unlabelled uncoloured $k$-tree together at the root-front. In order to handle this, we use colours. 
Consider the set of all front-colourings of $\mathcal{M}$-objects such that the root-front receives colour $k+1$. Note that each branch in a coloured $\mathcal{M}$-object is coloured identically, as fronts that are mirror to each other receive the same colour. Hence a coloured $\mathcal{M}$-object is constructed out of copies of a single coloured $\bar{B}^{{ }_{\mathrm{w}}}$-object. For each cycle type $\mu \vdash k$ let $\left(\bar{B}^{\mathrm{o}_{\mathrm{w}}}\right)_{\mu}(z)$ denote the generating series of the class of all colourings of $\bar{B}^{\circ}{ }^{\mathrm{w}}$-objects such that the root-front receives colour $k+1$ and such that the object is invariant under recolouring by a fixed permutation of degree $k$ with cycle type $\mu$. Likewise, we let $M_{\mu}(z)$ count $\mu$-invariant coloured $\mathcal{M}$-objects. Then

$$
M_{\mu}(z)=\sum_{\ell \geqslant 2}\left(\bar{B}^{\circ \mathrm{w}}\right)_{\mu}\left(z^{\ell}\right)
$$

Furthermore, we have the following substraction-free decomposition of $\mathcal{V}^{(3), \text { dec }}$.

Lemma 5.3 It holds that

$$
V^{(3), \operatorname{dec}}(z)=\sum_{\mu \vdash k} z_{\mu}^{-1} C_{\mu}(z) \sum_{\ell \geqslant 2}\left(\bar{B}^{\circ_{w}}\right)_{\mu}\left(z^{\ell}\right) .
$$

For each $\mu \vdash k$, the series $M_{\mu}(z)=\sum_{\ell \geqslant 2}\left(\bar{B}^{\circ_{w}}\right)_{\mu}\left(z^{\ell}\right)$ has radius of convergence strictly larger than $\rho_{k}$.

Proof. It is easy to see that $\left(\bar{B}^{\circ_{\mathrm{w}}}\right)_{\mu}(z)$ and $\bar{B}_{\mu}(z)$ have the same radius of convergence, because any $\bar{B}_{\mu}$-object with $n$ hedra corresponds to at least 1 and at most $n k$ ! objects from $\left(\bar{B}^{\circ}\right)_{\mu}$. By Lemma 4.1 it follows that $M_{\mu}(z)$ has radius of convergence strictly larger than $\rho_{k}$. Now, consider the class of colourings of $\mathcal{V}^{(3) \text {,dec }}$-objects where again the root-front is required to receive colour $k+1$. Applying Burnside's lemma (Lemma 3.1) yields (5.7).

\subsection{Conclusion}

The generating series we derived in equations (5.2), (5.4) and (5.7) may be summarized as follows.

Theorem 5.4 It holds that

$$
z U^{\prime}(z)=B(z)+V^{(2)}(z)+V^{(3), \operatorname{sym}}(z)+\sum_{\mu \vdash k} z_{\mu}^{-1} C_{\mu}(z) \sum_{\ell \geqslant 2}\left(\bar{B}^{\mathrm{o}_{w}}\right)_{\mu}\left(z^{\ell}\right) .
$$

This is a substraction-free alternative to the dissymmetry equation [14, Lemma 6].

Note that, using (5.8), we are able to retrieve the asymptotic number of unlabelled $k$-trees with $n$ hedra (see (5.9)) which was proved in [7, Theorem 3] using a different approach.

Since the series $V^{(2)}(z), V^{(3), s y m}(z)$ and

$$
\sum_{\mu \vdash k, \mu \neq 1^{k}} z_{\mu}^{-1} \bar{B}_{\mu}(z) \sum_{\ell \geqslant 2}\left(\bar{B}^{\mathrm{ow}_{\mathrm{w}}}\right)_{\mu}\left(z^{\ell}\right)
$$

have radius of convergence strictly larger than $\rho_{k}$, and $\bar{B}_{1^{k}}(z)$ has a dominant singularity of squareroot type at $\rho_{k}$, we may apply equations (3.9) and (4.1) together with general principles (e.g. [29, Lemma 3.2]) to deduce that

$$
n\left[z^{n}\right] U(z) \sim \frac{\left(k \rho_{k}\right)^{-k}}{k k !}\left(1+k \sum_{\ell \geqslant 2}\left(\bar{B}^{\circ_{w}}\right)_{1^{k}}\left(\rho_{k}^{\ell}\right)\right)\left[z^{n}\right] \bar{B}_{1^{k+1}}(z) .
$$


In general the operations of cycle-pointing and colouring $k$-trees do not commute. However, colouring 'well' pointed $k$-trees is the same as cycle-pointing coloured $k$-trees. That is,

$$
\left(\bar{B}^{\circ}\right)_{1^{k}}(z)=z \bar{B}_{1^{k}}^{\prime}(z) .
$$

Note that there is an asymptotic expansion

$$
\left[z^{n}\right] \bar{B}_{1^{k}}(z) \sim \sqrt{\frac{1+k \sum_{\ell \geqslant 2} \bar{B}_{1^{k}}^{\prime}\left(\rho_{k}^{\ell}\right) \rho_{k}^{\ell}}{2 \pi k^{2}}} n^{-3 / 2} \rho_{k}^{-n}
$$

that may be deduced from equation (4.1) using [3, Theorem 28]. Hence

$$
\left[z^{n}\right] U(z) \sim \frac{\left(k \rho_{k}\right)^{-k}}{k^{2} k ! \sqrt{2 \pi}}\left(1+k \sum_{\ell \geqslant 2}\left(\bar{B}^{\circ_{w}}\right)_{1^{k}}\left(\rho_{k}^{\ell}\right)\right)^{3 / 2} n^{-5 / 2} \rho_{k}^{-n} .
$$

\section{Proof of the main theorems}

In order to sample an unlabelled $k$-tree with $n$ hedra uniformly at random, we may uniformly select a cycle-pointed $k$-tree from $\mathcal{V}_{n}$ and then forget about the marked cycle. The decomposition in (5.1) allows us to divide the study of $\mathcal{V}_{n}$ into three cases, depending on the cycle-centre. In the following, we treat each part individually.

\subsection{Hedron-rooted $k$-trees}

As noted in equation (5.2), $\mathcal{V}^{(1)}$-objects are exactly unlabelled uncoloured hedron-rooted $k$-trees.

Lemma 6.1 Theorems 2.1, 2.2 and 2.3 hold for the uniform random unlabelled uncoloured hedronrooted $k$-tree with $n$ hedra.

Proof. Recall that for any cycle type $\lambda \vdash k+1$ we fixed a permutation $\pi_{\lambda} \in \mathfrak{S}_{k+1}$ with type $\lambda$. By Lemma 3.2 it follows that in order to uniformly sample an unlabelled hedron-marked $k$-tree with $n$ hedra we may proceed in two steps. First we draw a cycle type $\lambda \vdash k+1$ at random with probability given by $z_{\lambda}^{-1}\left(\left[z^{n}\right] B(z)\right)^{-1}\left[z^{n}\right] B_{\lambda}(z)$. In the second step we uniformly choose a front-coloured $k$ tree with $n$ hedra that is fixed by the permutation $\pi_{\lambda}$. By Lemma 4.1 and equation (3.10) the cycle type is exponentially likely to be equal to $1^{k+1}$. The special case $B_{1^{k+1}}(z)=z C_{1^{k}}(z)^{k+1}$ of equation (3.10) corresponds to the fact that any $B_{1^{k+1}}$-object may be constructed in a canonical way by gluing the root-fronts of $k+1 C_{1^{k}}$-objects together to form a root-hedron. (See Section 3.3 for details.) By Lemma 4.1 it holds that $\left[z^{n}\right] C_{1^{k}}(z) \sim \rho_{k}^{-n} n^{-3 / 2} c_{1^{k}}\left(1+O\left(n^{-1}\right)\right)$ for some constant $c_{1^{k}}>0$. Hence, either by direct calculations or by applying more general principles of random partitions $[26,29]$, it follows that the largest $C_{1^{k}}$-component in a random $B_{1^{k+1}}$-object of size $n$ has size $n-O(1)$. By Lemma 4.2 it follows that the limits of Theorem 2.1 and Theorem 2.3 hold for the largest $C_{1^{k}}$-component.

After rescaling edge-lengths by $\Theta\left(n^{-1 / 2}\right)$, the small components glued to the giant $C_{1^{k^{-}}}$ component contract to a single point. Hence, using the definition of the Gromov-HausdorffProkhorov distance in (2.1), it follows that the Gromov-Hausdorff-Prokhorov scaling limit for

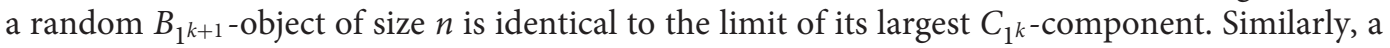
uniformly selected vertex of a random $B_{1^{k+1}}$-object of size $n$ lies with high probability in its largest $C_{1^{k}}$-component, since the total number of vertices in the combined small components is stochastically bounded. Moreover, for any fixed integer $r \geqslant 1$ the $r$-neighbourhood of that random vertex with high probability does not contain any of the vertices belonging to the root front. In fact, the Gromov-Hausdorff-Prokhorov convergence of the giant component entails that its distance 
from the root front has order $\sqrt{n}$. Hence, expressed in less technical terms, a myopic random vertex, that can only see other vertices with distance at most $r$, observes with high probability only vertices from the giant $C_{1}{ }^{k}$-component. Using the characterization of Benjamini-Schramm convergence in (2.12), it follows that the Benjamini-Schramm limit of a random $B_{1^{k+1}}$-object of size $n$ is identical to the Benjamini-Schramm limit of its largest $C_{1}$-component. We have thus verified that the limits of Theorem 2.1 and Theorem 2.3 both hold for random $B_{1^{k+1}}$-object of size $n$ as $n$ tends to infinity.

Since the cycle type in our sampling procedure is with high probability equal to $1^{k+1}$, it follows that the limits of Theorem 2.1 and Theorem 2.3 both hold for random hedron-rooted unlabelled $k$-trees, with the scaling constant of the scaling limit being equal to those for the case of unlabelled $k$-trees rooted at a front of distinguishable vertices.

It remains to prove the tail bound for the diameter. By Lemma 4.2 there are constants $C, c>0$ such that the probability of the $k$-tree diameter of a $C_{1}$-object of size $n$ being larger than $x \geqslant 0$ is bounded uniformly by $C \exp \left(-c x^{2} / n\right)$. An $n$-sized $B_{1} k+1$-object consists of $k+1$ components whose sizes (number of hedra) $n_{1}, \ldots, n_{k+1}$ sum up to $n-1$, since none of them contain the root hedron. Let $\mathrm{B}_{n}$ be a uniformly selected unlabelled, hedron-rooted and front-coloured $k$-tree with $n$ vertices and let $C^{1}\left(\mathrm{~B}_{n}\right), \ldots, C^{k+1}\left(\mathrm{~B}_{n}\right)$ denote its components. If $\mathrm{B}_{n}$ has diameter at least $x$, then at least one of its components has diameter at least $x / 2$. Letting $\left|C^{i}\left(\mathrm{~B}_{n}\right)\right|$ denote the number of hedra in the component $C^{i}\left(B_{n}\right)$, it follows that

$$
\begin{aligned}
\mathbb{P}\left(\mathrm{D}\left(\mathrm{B}_{n}\right) \geqslant x\right) & \leqslant \sum_{n_{1}+\cdots+n_{k+1}=n-1} \mathbb{P}\left(\left|C^{i}\left(\mathrm{~B}_{n}\right)\right|=n_{i}, i=1, \ldots, k+1\right) \sum_{i=1}^{k+1} C \exp \left(-c x^{2} /\left(4 n_{i}\right)\right) \\
& \leqslant C(k+1) \exp \left(-c x^{2} /(4 n)\right) .
\end{aligned}
$$

We argued above that the total variation distance of uniform random coloured and uniform random uncoloured hedron-rooted $k$-trees (i.e. $B_{1^{k+1}}$-objects and $B$-objects) with $n$ vertices is exponentially small (as the partition type we considered is exponentially likely to be equal to $1^{k+1}$ ). It follows that the tail bound for the diameter in Theorem 2.2 holds for the random unlabelled uncoloured hedron-rooted $k$-tree with $n$ hedra. This concludes the proof.

\subsection{Cycle-pointed $k$-trees with a hedron cycle-centre}

Lemma 5.1 readily implies that we can safely neglect the case of a hedron cycle-centre.

Lemma 6.2 A uniformly selected cycle-pointed $k$-tree from the class $\mathcal{V}_{n}$ is exponentially unlikely to have a hedron as the cycle-centre.

\subsection{Cycle-pointed $k$-trees with a front cycle-centre}

Recall that we split the class $\mathcal{V}^{(3)}$ into $\mathcal{V}^{(3) \text {,sym }}$ and $\mathcal{V}^{(3) \text {,dec }}$. Lemma 5.2 and the enumerative observations in Section 5.4 readily entail that we may safely neglect the class $\mathcal{V}^{(3) \text { sym }}$.

Lemma 6.3 A uniformly selected cycle-pointed $k$-tree from the class $\mathcal{V}_{n}^{(3)}$ is exponentially unlikely to belong to $\mathcal{V}_{n}^{(3), \text { sym }}$.

It remains to prove the main theorems for random elements from $\mathcal{V}_{n}^{(3) \text {,dec }}$. 
Lemma 6.4 Theorems 2.1, 2.2 and 2.3 hold for the uniformly selected cycle-pointed $k$-tree from the class $\mathcal{V}_{n}^{(3), \mathrm{dec}}$.

Proof. Recall the substraction-free decomposition given in equation (5.7). Using Lemma 4.1 and Lemma 5.3 it follows that all summands with $\mu \neq 1^{k}$ have radius of convergence strictly larger than $\rho_{k}$. The summand for $\mu=1^{k}$ represents pairs of a $C_{1^{k}}$-object (that bijectively corresponds to an unlabelled uncoloured $k$-tree rooted at a front of distinguishable vertices: see Section 4) and an

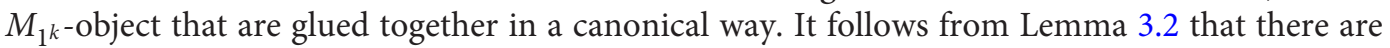
constants $C, c>0$ such that the total variation distance between the uniform measure on $\mathcal{V}_{n}^{(3)}$ and the uniform measure on $n$-hedron unlabelled, uncoloured, cycle-pointed $k$-trees obtained from $\left(C_{1^{k}}, M_{1^{k}}\right)$-pairs is bounded by $C \exp (-c n)$ for all $n$. Since $M_{1^{k}}(z)$ has radius of convergence strictly larger than $\rho_{k}$, it follows easily from the asymptotic expansion of $\left[z^{n}\right] C_{1^{k}}(z)$ (both the dominant term and the second order term) that the marked part has stochastically bounded size as $n$ becomes large.

Arguing analogously as in the proof of Lemma 6.1, it follows from Lemma 4.2 that Theorems 2.1, 2.2 and 2.3 hold for the uniformly selected cycle-pointed $k$-tree from the class $\mathcal{V}_{n}^{(3), \mathrm{dec}}$.

\subsection{Proof of Theorems 2.1, 2.2 and 2.3}

Suppose that we uniformly select a cycle-pointed $k$-tree $\mathrm{V}_{n}$ from $\mathcal{V}_{n}$. Lemma 6.2 entails that $\mathrm{V}_{n}$ is exponentially unlikely to belong to $\mathcal{V}_{n}^{(2)}$. Lemma 6.1 entails that Theorems $2.1,2.2$ and 2.3 hold for the random graph obtained by conditioning $\mathrm{V}_{n}$ to belong to $\mathcal{V}_{n}^{(1)}$. Lemma 6.3 and Lemma 6.4 entail that this is also the case when $\mathrm{V}_{n}$ is conditioned to belong to $\mathcal{V}_{n}^{(3)}$. As $\mathrm{U}_{n}$ is obtained from $\mathrm{V}_{n}$ by forgetting about the marked cycle, this proves Theorems 2.1, 2.2 and 2.3.

\section{Acknowledgement}

We are grateful to the referees for suggestions leading to an improved presentation.

\section{References}

[1] Aldous, D. (1991) The continuum random tree, II: An overview. In Stochastic Analysis (Durham, 1990), Vol. 167 of London Mathematical Society Lecture Note Series, Cambridge University Press, pp. 23-70.

[2] Beineke, L. W. and Pippert, R. E. (1969) The number of labeled k-dimensional trees. J. Combin. Theory $6200-205$.

[3] Bell, J. P., Burris, S. N. and Yeats, K. A. (2006) Counting rooted trees: The universal law $t(n) \sim C \rho^{-n} n^{-3 / 2}$. Electron. J. Combin. 13 R63.

[4] Bergeron, F., Labelle, G. and Leroux, P. (1998) Combinatorial Species and Tree-Like Structures, Vol. 67 of Encyclopedia of Mathematics and its Applications, Cambridge University Press.

[5] Bodirsky, M., Fusy, É., Kang, M. and Vigerske, S. (2011) Boltzmann samplers, Pólya theory, and cycle pointing. SIAM J. Comput. 40 721-769.

[6] Darrasse, A. and Soria, M. (2009) Limiting distribution for distances in k-trees. In Combinatorial Algorithms (IWOCA 2009) (J. Fiala et al., eds), Vol. 5874 of Lecture Notes in Computer Science, Springer, pp. 170-182.

[7] Drmota, M. and Jin, E. Y. (2014) An asymptotic analysis of labeled and unlabeled $k$-trees. Algorithmica $75579-605$.

[8] Drmota, M., Jin, E. Y. and Stufler, B. (2019) Graph limits of random graphs from a subset of connected $k$-trees. Random Struct. Alg. 55 125-558.

[9] Durrett, R. T. and Iglehart, D. L. (1977) Functionals of Brownian meander and Brownian excursion. Ann. Probab. 5 130-135.

[10] Flajolet, P. and Sedgewick, R. (2010) Analytic Combinatorics, Cambridge University Press.

[11] Foata, D. (1971) Enumerating k-trees. Discrete Math. 1 181-186.

[12] Fowler, T., Gessel, I., Labelle, G. and Leroux, P. (2002) The specification of 2-trees. Adv. Appl. Math. 28 145-168. 
[13] Gainer-Dewar, A. (2012) $\Gamma$-species and the enumeration of $k$-trees. Electron. J. Combin. 19 P45.

[14] Gainer-Dewar, A. and Gessel, I. M. (2014) Counting unlabeled k-trees. J. Combin. Theory Ser. A 126 177-193.

[15] Harary, F. and Palmer, E. M. (1968) On acyclic simplicial complexes. Mathematika 15 115-122.

[16] Harary, F. and Palmer, E. M. (1973) Graphical Enumeration, Academic Press.

[17] Iriza, A. D. (2015) Enumeration and random generation of unlabeled classes of graphs: A practical study of cyclepointing and the dissymmetry theorem. Master's thesis, Princeton University.

[18] Joyal, A. (1981) Une théorie combinatoire des séries formelles. Adv. Math. 42 1-82.

[19] Miermont, G. (2009) Tessellations of random maps of arbitrary genus. Ann. Sci. Éc. Norm. Supér. 42 725-781.

[20] Moon, J. W. (1969) The number of labeled k-trees. J. Combin. Theory Ser. A 6 196-199.

[21] Otter, R. (1948) The number of trees. Ann. of Math. (2) $49583-599$.

[22] Pólya, G. (1937) Kombinatorische Anzahlbestimmungen für Gruppen, Graphen und chemische Verbindungen. Acta Math. 68 145-254.

[23] Stufler, B. (2016) Limits of random tree-like discrete structures. arXiv:1612.02580

[24] Stufler, B. (2017) Asymptotic properties of random unlabelled block-weighted graphs. arXiv:1712.01301

[25] Stufler, B. (2017) Scaling limits of random outerplanar maps with independent link-weights. Ann. Inst. H. Poincaré Probab. Statist. 53 900-915.

[26] Stufler, B. (2018) Gibbs partitions: The convergent case. Random Struct. Alg. 53 537-558.

[27] Stufler, B. (2018) Random enriched trees with applications to random graphs. Electron. J. Combin. 25 P3.11.

[28] Stufler, B. (2019) The continuum random tree is the scaling limit of unlabelled unrooted trees. Random Struct. Alg. 55 496-528.

[29] Stufler, B. (2020) Unlabelled Gibbs partitions. Combin. Probab. Comput. 29 293-309.

Cite this article: Jin EY and Stufler B (2020). Graph limits of random unlabelled $k$-trees. Combinatorics, Probability and Computing 29, 722-746. https://doi.org/10.1017/S0963548320000164 